\title{
Three-dimensional time and frequency-domain theory of femtosecond $x$-ray pulse generation through Thomson scattering
}

\author{
Winthrop J. Brown and Frederic V. Hartemann \\ Lawrence Livermore National Laboratory, Livermore, California 94550, USA
}

(Received 30 January 2004; published 11 June 2004)

\begin{abstract}
The generation of high intensity, ultrashort $\mathrm{x}$-ray pulses enables exciting new experimental capabilities, such as femtosecond pump-probe experiments used to temporally resolve material structural dynamics on atomic time scales. Thomson backscattering of a high intensity laser pulse with a bright relativistic electron bunch is a promising method for producing such high-brightness $\mathrm{X}$-ray pulses in the 10-100 keV range within a compact facility. While a variety of methods for producing subpicosecond $\mathrm{x}$-ray bursts by Thomson scattering exist, including compression of the electron bunch to subpicosecond bunch lengths and/or colliding a subpicosecond laser pulse in a side-on geometry to minimize the interaction time, a promising alternative approach to achieving this goal while maintaining ultrahigh brightness is the production of a time-correlated (or chirped) $\mathrm{x}$-ray pulse in conjunction with pulse slicing or compression. We present the results of a complete analysis of this process using a recently developed 3D time and frequency-domain code for analyzing the spatial, temporal, and spectral properties an x-ray beam produced by relativistic Thomson scattering. Based on the relativistic differential cross section, this code has the capability to calculate time and space dependent spectra of the x-ray photons produced from linear Thomson scattering for both bandwidth-limited and chirped incident laser pulses. Spectral broadening of the scattered $\mathrm{x}$-ray pulse resulting from the incident laser bandwidth, laser focus, and the transverse and longitudinal phase space of the electron beam were examined. Simulations of chirped $\mathrm{x}$-ray pulse production using both a chirped electron beam and a chirped laser pulse are presented. Required electron beam and laser parameters are summarized by investigating the effects of beam emittance, energy spread, and laser bandwidth on the scattered x-ray spectrum. It is shown that sufficient temporal correlation in the scattered $\mathrm{x}$-ray spectrum to produce sub-100 fs temporal slice resolution can be produced from state-of-the-art, high-brightness electron beams without the need to perform longitudinal compression on the electron bunch.
\end{abstract}

DOI: 10.1103/PhysRevSTAB.7.060703

PACS numbers: 41.60.-m, 52.59.-f, 52.38.-r

\section{INTRODUCTION}

The use of short laser pulses to generate high intensity, ultrashort $\mathrm{x}$-ray pulses enables exciting new experimental capabilities, such as femtosecond pump-probe experiments used to temporally resolve the structural dynamics of high- $Z$ materials on atomic (femtosecond) time scales $[1,2]$. In particular the unique conditions of atomicscale interaction have led to a recent experimental push to develop high-brightness, femtosecond, hard $\mathrm{x}$-ray sources. The energy levels relevant to the innershell electron properties, which are responsible for the most fundamental atomic-scale effects, require photon energies well above those generated by modern ultrafast laser systems. Furthermore, the short time scales associated with atomic motion, tens to hundreds of femtoseconds, require shorter pulses than those provided by synchrotron-based $\mathrm{x}$-ray sources. The development of a system capable of making measurements on these scales would open up regions of currently underexplored science, such as phase transitions in materials under shock loading and chemical reaction dynamics. The most promising methods for generating tunable, subpicosecond, very high-brightness electromagnetic radiation at short wavelengths $(<1 \AA)$ rely on either coherent radiation produced by an $x$-ray free-electron laser (FEL), such as the planned Linac Coherent Light Source [3], or incoherent production through relativistic Thomson scattering, which has previously been employed for time resolved diffraction measurements at LBNL [4,5] and is currently being investigated at several laboratories around the world [6-11]. Additionally, a growing number of research groups worldwide are exploring different $\mathrm{x}$-ray production mechanisms such as ultrafast, laser driven $K_{\alpha}$ sources [12] and electron bunch slicing in synchrotrons [13]. While coherent radiation sources generate higher power and narrower spectral bandwidths when compared to incoherent scattering, the Thomson source's potential for high peak brightness with a relatively compact and affordable system makes it an attractive alternative for many applications.

In this paper, we employ a newly developed threedimensional (3D) time and frequency-domain code to examine in detail the use of Thomson scattering as a means of producing high-brightness, femtosecond $\mathrm{x}$-ray pulses, and, in particular, the production of chirped $\mathrm{x}$-ray pulses. The ability to produce time-correlated (chirped) $\mathrm{x}$-ray spectra within a single pulse would be desirable for ultimately improving the resolution of pump-probe time resolved diffraction experiments. It has previously been proposed, for example, that chirped $\mathrm{x}$-ray pulses could be 
used to obtain a series of time-correlated measurements within a single Laue diffraction pattern, while eliminating the relevance of the timing jitter between the pump and probe pulses [14]. Slicing of chirped $x$-ray pulses has also been proposed as a method of seeding very short pulses into a two-stage x-ray FEL [15] to produce very high-brightness, ultrashort pulses. In addition, strained crystal [16] or grating compressor [17] pulse compression schemes could be used to compress a chirped $\mathrm{x}$-ray pulse without suffering the photon losses inherent in pulse slicing.

While the theory of Thomson backscattered radiation is well known and has been well documented [18-24], there remains a need to have a complete three-dimensional time resolved computational capability for the full determination of the temporally and spatially resolved spectra and intensity distributions produced from a Thomson interaction of arbitrary geometry. This capability is crucial for both the design of Thomson scattered $\mathrm{x}$-ray sources, as well as future experiments and applications utilizing such sources. In this paper, we present a newly developed fully three-dimensional time and frequency-domain code used for calculations of Thomson backscattering of a short, intense laser pulse with a relativistic electron bunch. This is accomplished by employing the Thomson differential cross section generalized for relativistic interactions, derived from the standard electron rest frame differential cross section and employing appropriate Lorentz transformations of the incident laser pulse into the rest frame, and of the scattered photons into the lab frame. The 3D code developed using this approach was designed to enhance existing computational capabilities, namely, a 3D frequency-domain code developed by Hartemann et al. [18]. In particular, the new code is well suited for analyzing time-dependent spectra of the $\mathrm{x}$ rays produced from linear Thomson scattering and hence is ideally suited for the study of chirped x-ray pulse production. To our knowledge, the analysis presented in this paper is the first detailed investigation of chirped $\mathrm{x}$-ray pulse production through Thomson scattering.

The remainder of this paper is divided into five sections. In Sec. II, a derivation of the relativistic differential cross section is presented for the case of a single photon incident on a single electron for arbitrary interaction geometries. While considering only a single electron and photon, the treatment is made as general as possible for easy inclusion of the $6 \mathrm{D}$ phase space of both the laser and the electron beams later in the paper. In Sec. III, a brief overview of the properties of Thomson backscattered radiation is presented, and simple approximate forms of the x-ray intensity angular distribution are introduced for clarity. In Sec. IV, the treatment is expanded to include the spectral broadening resulting from the finite laser bandwidth, perpendicular $k$-vector distribution resulting from the finite laser focus, the electron beam energy spread, and the finite electron beam emit- tance. In Sec. V, an overview of the time and frequencydomain code developed from the theory presented in Secs. II, III, and IV is presented. Finally, in Sec. VI, simulations of chirped $\mathrm{x}$-ray pulse production and slicing are presented. Two different methods for the chirped $\mathrm{x}-$ ray pulse production are contrasted: (1) collision of a chirped electron bunch with a bandwidth-limited laser pulse in a head-on geometry and (2) collision of a monochromatic electron bunch with a chirped laser pulse in a side-on geometry. It is shown that sub-100 fs x-ray pulses can be produced utilizing pulse slicing techniques, showing promise as a powerful method for achieving atomic time scale resolution in dynamic diffraction experiments. In a companion paper, the 3D theory is benchmarked against experimental data obtained at LLNL's PLEIADES facility.

\section{INTRODUCTION TO THOMSON SCATTERING}

Thomson backscattered photons are produced when an electron beam collides with a photon beam (i.e., laser). In the Thomson limit, the incident photon has a very small energy compared to the electron rest mass, and hence, the scattered photon has the same energy as the incident photon in the electron rest frame. For the case of an electron distribution at rest, the total number of scattered photons per unit time is simply the overlap integral of the product of the total Thomson cross section multiplied by the flux of incident photons, leading to

$$
N_{s}^{\prime}=\int \operatorname{con}_{e}^{\prime}(\mathbf{r}, t) n_{\gamma}^{\prime}(\mathbf{r}, t) d^{3} \mathbf{r} d t
$$

where $c$ is the speed of light, $\sigma$ is the total Thomson cross section, and $n_{\gamma}^{\prime}(\mathbf{r}, t)$ and $n_{e}^{\prime}(\mathbf{r}, t)$ are the photon and electron density in the electron beam rest frame, respectively. To generalize this for a relativistic electron beam, it can be noted that the total number of scattered photons is Lorentz invariant, and that the above expression can be expressed covariantly as the integration of the product of the electron four current, $J^{\mu}=e c n_{e}\left(1, \boldsymbol{\beta}_{e}\right)$, and the photon four flux, $\Phi^{\mu}=c n_{\gamma}(1, c \mathbf{k} / \omega)$ resulting in [19]

$$
\begin{aligned}
N_{s} & =\frac{\sigma}{c e} \int J^{\mu} \Phi_{\mu} d^{4} x \\
& =\sigma c \int\left(1-\boldsymbol{\beta}_{e} \cdot \mathbf{k} \frac{c}{\omega}\right) n_{e}(\mathbf{r}, t) n_{\gamma}(\mathbf{r}, t) d^{3} \mathbf{r} d t
\end{aligned}
$$

For scattering from a single electron, $n_{e}(\mathbf{r}, t)=$ $\delta\left[\mathbf{r}_{e}(t)\right]$, where $\mathbf{r}_{e}(t)$ is the position of the electron at time $t$. Thus, the rate of scattered photons from a single electron becomes

$$
\frac{d N_{s}}{d t}=\sigma c\left(1-\boldsymbol{\beta}_{e} \cdot \mathbf{k} \frac{c}{\omega}\right) n_{\gamma}\left[\mathbf{r}_{e}(t), t\right] .
$$

Likewise, the rate of photons scattered into a given solid angle is given by 


$$
\frac{d N_{s}}{d \Omega d t}=c\left(1-\boldsymbol{\beta}_{e} \cdot \mathbf{k} \frac{c}{\omega}\right) n_{\gamma}\left(\mathbf{r}_{e}, t\right) \frac{d \sigma}{d \Omega}
$$

while the rate scattered per unit frequency is given by

$$
\begin{aligned}
\frac{d N_{s}}{d \omega_{s} d \Omega d t} & =c\left(1-\boldsymbol{\beta}_{e} \cdot \mathbf{k} \frac{c}{\omega}\right) n_{\gamma} \frac{d \sigma}{d \Omega d \omega_{s}} \\
& =c\left(1-\boldsymbol{\beta}_{e} \cdot \mathbf{k} \frac{c}{\omega}\right) n_{\gamma}\left(\mathbf{r}_{e}, t\right) \frac{d \sigma}{d \Omega} \delta\left[\omega_{s}-\omega g(\theta)\right],
\end{aligned}
$$

where $d \sigma / d \Omega$ is the differential cross section for Thomson scattering, $\omega_{s}$ is the angular frequency of the scattered photon, and $g(\theta)$ is the relativistic Doppler upshift of the scattered photon, which is dependent on both the angle, $\theta$, between the observation direction and the electron direction, as well as the angle between the electron direction and the incident photon. Equation (5) describes the complete temporal, spectral, and spatial properties of the scattered $\mathrm{x}$-ray distribution and is the basis for the time and frequency-domain code presented in this paper. The validity of Eq. (5) requires the scattering remain linear, which means the normalized vector potential within the laser pulse, given by $a_{0}=e A / m c$, where $m$ is the rest mass of the electron, is much less than unity. In addition, it is assumed there is no recoil of the electron, implying the incident photon energy in the electron's rest frame is much less than the electron rest mass. It is also noted that the calculations presented in this paper assume the background motion of each electron through the incident laser pulse is ballistic, which is a good approximation provided the two above conditions are met, and the plasma oscillation period $\left(1 / \omega_{p}\right)$ of the electron beam is much longer than the interaction time, which implies that space-charge effects can be neglected.

\section{A. Derivation of rest frame differential cross section}

A general expression for the differential cross section in Eq. (5) can be derived by first transforming the wave vector of the incident photon into the electron's rest frame. The corresponding rest frame differential cross section can then be transformed back into the lab frame. We begin by considering the rest frame as depicted in Fig. 1. If we represent the incident laser polarization vector as $\boldsymbol{\alpha}^{\prime}$, then the differential cross section is given by [25]

$$
\frac{d \sigma}{d \Omega^{\prime}}=r_{0}^{2}\left|\boldsymbol{\eta}^{\prime} \cdot \boldsymbol{\alpha}^{\prime}\right|^{2}
$$

where $\boldsymbol{\eta}^{\prime}$ is the scattered photon polarization vector, and $r_{0}$ is the classical electron radius. $\boldsymbol{\eta}^{\prime}$ can be separated into two perpendicular components. One, $\boldsymbol{\eta}_{1}^{\prime}$, is in the plane defined by the observation vector, $\boldsymbol{n}^{\prime}$, and photon wave vector $\mathbf{k}_{0}^{\prime}$. The other, $\boldsymbol{\eta}_{2}^{\prime}$, is in the plane perpendicular to both $\mathbf{k}_{0}^{\prime}$ and $\boldsymbol{\eta}_{1}^{\prime}$, where [25]

$$
\boldsymbol{\eta}_{1}^{\prime}=\cos \theta_{e}^{\prime}\left(\mathbf{e}_{x}^{\prime} \cos \phi_{e}^{\prime}+\mathbf{e}_{y}^{\prime} \sin \phi_{e}^{\prime}\right)-\mathbf{e}_{z}^{\prime} \sin \theta_{e}^{\prime}
$$

and

$$
\boldsymbol{\eta}_{2}^{\prime}=-\mathbf{e}_{x}^{\prime} \sin \phi_{e}^{\prime}+\mathbf{e}_{y}^{\prime} \cos \phi_{e}^{\prime}
$$

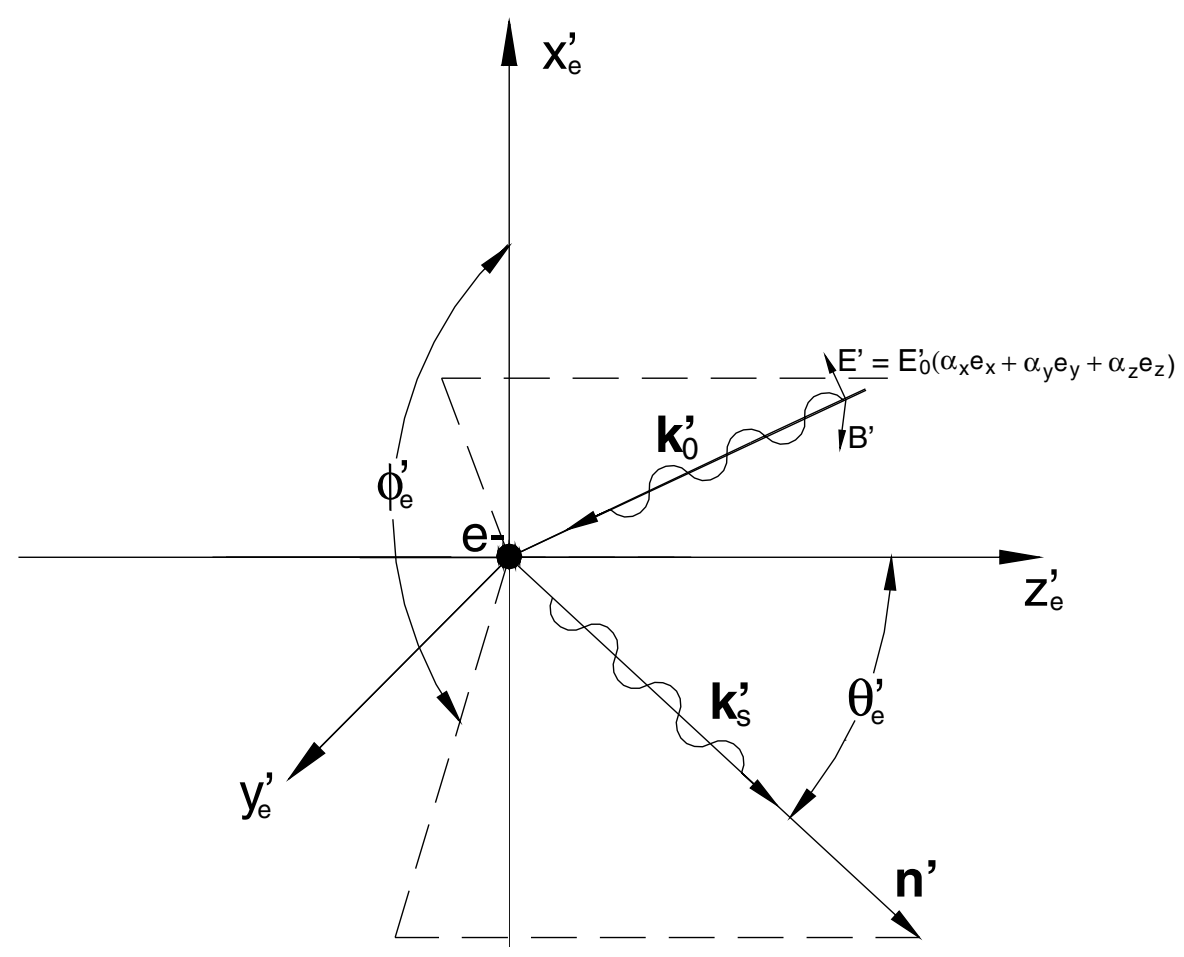

FIG. 1. Thomson interaction geometry in the electron rest frame. 
(a)

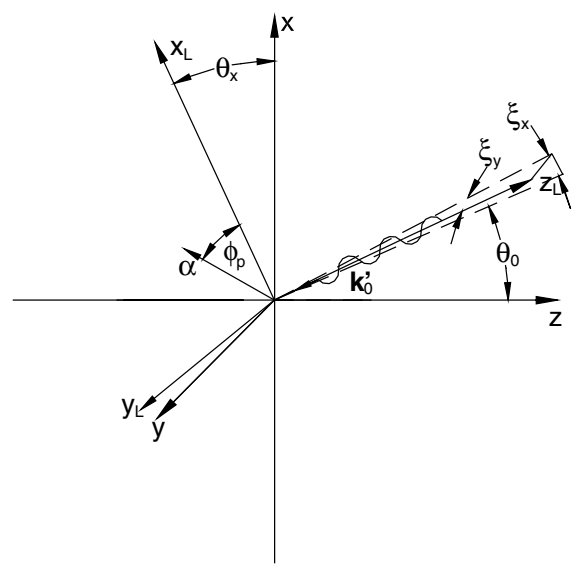

(b)

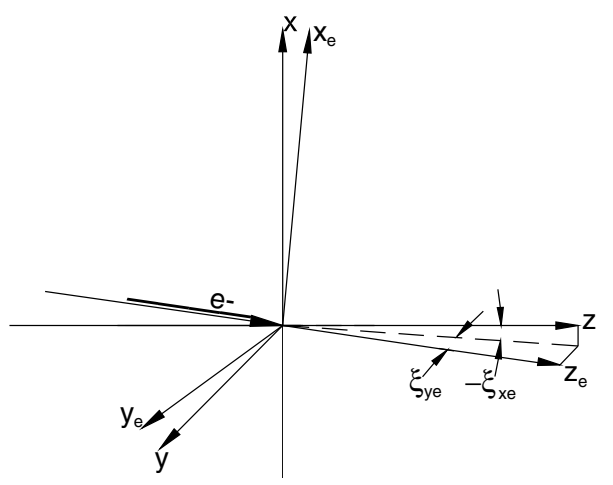

FIG. 2. (a) Illustration of laser incident direction and polarization. $\boldsymbol{\alpha}$ is the direction of the polarization vector of the laser, where $\phi_{p}$ represents the rotation angle of $\boldsymbol{\alpha}$ about the $z_{L}$ axis. (b) Illustration of the electron incident direction. The electron beam is incident along the $z$ axis, but the direction of each electron deviated by the angles specified by $\xi_{x e}$ and $\xi_{y e}$.

Using Eqs. (6)-(8) and summing over final polarization states yields the nonrelativistic Thomson cross section for an arbitrary linearly polarized incident photon, resulting in

$$
\begin{aligned}
\frac{1}{r_{0}^{2}} \frac{d \sigma}{d \Omega^{\prime}}= & \alpha_{x}^{\prime 2}\left(1-\cos ^{2} \phi_{e}^{\prime} \sin ^{2} \theta_{e}^{\prime}\right)+\alpha_{y}^{\prime 2}\left(1-\sin ^{2} \phi_{e}^{\prime} \sin ^{2} \theta_{e}^{\prime}\right) \\
& +\alpha_{z}^{\prime 2}\left(1-\cos ^{2} \theta_{e}^{\prime}\right) \\
& -2 \alpha_{x}^{\prime} \alpha_{y}^{\prime}\left(\cos \phi_{e}^{\prime} \sin \theta_{e}^{\prime}\right)\left(\sin \phi_{e}^{\prime} \sin \theta_{e}^{\prime}\right) \\
& -2 \alpha_{x}^{\prime} \alpha_{z}^{\prime} \cos \theta_{e}^{\prime}\left(\cos \phi_{e}^{\prime} \sin \theta_{e}^{\prime}\right) \\
& -2 \alpha_{x}^{\prime} \alpha_{z}^{\prime} \cos \theta_{e}^{\prime}\left(\sin \phi_{e}^{\prime} \sin \theta_{e}^{\prime}\right) .
\end{aligned}
$$

To make Eq. (9) practical for relativistic beams, it is desirable to express the components of the rest frame incident laser polarization vector in lab frame coordinates. We consider a stationary lab frame $(x, y, z)$ in which an electron beam is traveling in the $+z$ direction and a laser beam is incident at an angle $\theta_{0}$ with respect to the $-z$ direction in the $x-z$ plane $\left(\theta_{0}=0\right.$ corresponds to a head-on collision). To generalize the interaction for arbitrary linear polarizations, the laser polarization vector is defined by its azimuthal angle $\phi_{p}$ about the laser wave vector $\mathbf{k}_{0}$, defined in a rotated coordinate system $\left(x_{L}, y_{L}\right.$, $z_{L}$ ), where $z_{L}$ is chosen to be antiparallel to the laser wave vector $\mathbf{k}_{0}$. For $\phi_{p}=0$, the laser is polarized in the $x-z$ plane.

To facilitate the inclusion of three-dimensional effects resulting from the electron and laser focus, the directions of the individual electrons and incident photon wave vectors are assumed to deviate slightly from the average directions defined above. As shown in Fig. 2(a), the direction of each incident photon wave vector will be specified by an additional rotation $\xi_{x}$ about the $y$ axis, and a rotation $\xi_{y}$ about the $x_{L}$ axis. Likewise, an electron lab frame $\left(x_{e}, y_{e}, z_{e}\right)$, defined such that the $z_{e}$ axis is collinear with the individual electron direction, will be specified by a rotation $\xi_{x e}$ about the $y$ axis and an angle $\xi_{y e}$ about the $x_{e}$ axis [Fig. 2(b)].

Further, to simplify the Lorentz transformations to and from the electron rest frame, our approach will be to first calculate the differential cross section in the electron lab frame and then rotate back to the stationary lab frame. We begin by calculating the components of the electric field from the laser polarization in the rotated electron lab frame. In the laser frame, the polarization vector has nonzero components in the $x_{L}$ and $y_{L}$ directions only such that

$$
\begin{aligned}
& E_{x L}=E_{0} \cos \phi_{p}, \quad E_{y L}=E_{0} \sin \phi_{p}, \\
& c B_{x L}=E_{0} \sin \phi_{p}, \quad c B_{y L}=-E_{0} \sin \phi_{p},
\end{aligned}
$$

where $E_{0}$ is the magnitude of the laser electric field. It is straightforward to perform the consecutive coordinate rotations to obtain these components in the electron lab frame, resulting in

$$
\mathbf{E}_{e}=\left(\mathbb{R}_{e y} \mathbb{R}_{e x} \mathbb{R}_{L x}^{-1} \mathbb{R}_{L y}^{-1}\right) \mathbf{E}_{L}
$$

where

$$
\mathbb{R}_{L x}^{-1} \mathbb{R}_{L y}^{-1}=\left(\begin{array}{ccc}
\cos \left(\theta_{0}+\xi_{x}\right) & -\sin \left(\theta_{0}+\xi_{x}\right) \sin \xi_{y} & \sin \left(\theta_{0}+\xi_{x}\right) \cos \xi_{y} \\
0 & \cos \xi_{y} & \sin \xi_{y} \\
-\sin \left(\theta_{0}+\xi_{x}\right) & -\sin \xi_{y} \cos \left(\theta_{0}+\xi_{x}\right) & \cos \left(\theta_{0}+\xi_{x}\right) \cos \xi_{y}
\end{array}\right)
$$

and 


$$
\mathbb{R}_{e y} \mathbb{R}_{e x}=\left(\begin{array}{ccc}
\cos \xi_{x e} & 0 & -\sin \xi_{x e} \\
-\sin \xi_{x e} \sin \xi_{y e} & \cos \xi_{y e} & -\cos \xi_{x e} \sin \xi_{y e} \\
\sin \xi_{x e} \cos \xi_{y e} & \sin \xi_{y e} & \cos \xi_{x e} \cos \xi_{y e}
\end{array}\right) .
$$

Defining $\theta_{x} \equiv \theta_{0}+\xi_{x}$, Eq. (11) leads to

$$
\begin{aligned}
& \frac{E_{x e}}{E_{0}}=\cos \phi_{p} \cos \left(\theta_{x}-\xi_{x e}\right)-E_{0} \sin \phi_{p} \sin \xi_{y} \sin \left(\theta_{x}-\xi_{x e}\right), \\
& \frac{E_{y e}}{E_{0}}=\cos \phi_{p} \sin \left(\theta_{x}-\xi_{x e}\right) \sin \xi_{y e}+\sin \phi_{p}\left[\cos \xi_{y} \cos \xi_{y e}+\sin \xi_{y} \sin \xi_{y e} \cos \left(\theta_{x}-\xi_{x e}\right)\right], \\
& \frac{E_{z e}}{E_{0}}=-\cos \phi_{p} \sin \left(\theta_{x}-\xi_{x e}\right) \cos \xi_{y e}-\sin \phi_{p}\left[\sin \xi_{y} \cos \xi_{y e} \cos \left(\theta_{x}-\xi_{x e}\right)-\cos \xi_{y} \sin \xi_{y e}\right],
\end{aligned}
$$

and correspondingly, for the magnetic field,

$$
\begin{aligned}
& \frac{c B_{x e}}{E_{0}}=\sin \phi_{p} \cos \left(\theta_{x}-\xi_{x e}\right)+\cos \phi_{p} \sin \xi_{y} \sin \left(\theta_{x}-\xi_{x e}\right), \\
& \frac{c B_{y e}}{E_{0}}=\sin \phi_{p} \sin \left(\theta_{x}-\xi_{x e}\right) \sin \xi_{y e}-\cos \phi_{p}\left[\cos \xi_{y} \cos \xi_{y e}+\sin \xi_{y} \sin \xi_{y e} \cos \left(\theta_{x}-\xi_{x e}\right)\right], \\
& \frac{c B_{z e}}{E_{0}}=-\sin \phi_{p} \sin \left(\theta_{x}-\xi_{x e}\right) \cos \xi_{y e}+\cos \phi_{p}\left[\sin \xi_{y} \cos \xi_{y e} \cos \left(\theta_{x}-\xi_{x e}\right)-\cos \xi_{y} \sin \xi_{y e}\right],
\end{aligned}
$$

where $E_{0}$ represents the magnitude of the incident electromagnetic wave.

The $\mathbf{E}$ and $\mathbf{B}$ fields can now be transformed to the electron rest frame to find the polarization vector $\boldsymbol{\alpha}^{\prime}$. Since the electron beam direction is defined to be in the $+z_{e}$ direction, this requires only a one-dimensional boost, leading to [25]

$$
\begin{aligned}
& E_{x}^{\prime}=\gamma\left(E_{x}-c \beta B_{y}\right), \quad E_{y}^{\prime}=\gamma\left(E_{y}+c \beta B_{x}\right), \\
& E_{z}^{\prime}=E_{z},
\end{aligned}
$$

where the prime denotes the electron rest frame. The rest frame polarization vector can now be calculated using the expression

$$
\alpha_{i}=\frac{E_{i}^{\prime}}{\left|E_{0}^{\prime}\right|}
$$

The normalization factor in Eq. (17) is easily determined by employing the invariance of the normalized vector potential magnitude $\left(a_{0}^{\prime}=a_{0}\right)$, leading to

$$
\frac{E_{0}}{E_{0}^{\prime}}=\frac{\omega_{0}^{\prime}}{\omega_{0}}=\gamma\left(1-\frac{c}{\omega_{0}} \boldsymbol{\beta} \cdot \mathbf{k}_{0}\right) \equiv g^{\prime}\left(\theta_{x}, \xi_{y}, \xi_{x e}, \xi_{y e}\right)
$$

where the transformation of the laser wavelength from the lab frame to the rest frame has been employed, and the quantity $g^{\prime}$ has been defined to be the ratio of the incident photon energy in the rest frame to the photon energy in the lab frame. In terms of the rotated electron and photon coordinate angles, $g^{\prime}$ is expressed as

$$
\begin{aligned}
g^{\prime}=\gamma\left[1+\beta\left\{\cos \left(\theta_{x}-\xi_{x e}\right) \cos \left(\xi_{y}-\xi_{y e}\right)\right.\right. \\
\left.\left.+\sin \xi_{y} \sin \xi_{y e}\left[1-\cos \left(\theta_{x}-\xi_{x e}\right)\right]\right\}\right] .
\end{aligned}
$$

Combining Eqs. (14), (15), and (17)-(19) results in the following expressions for the laser polarization vector in the electron's rest frame:

$$
\begin{aligned}
\alpha_{x e}^{\prime}=\frac{1}{g^{\prime}}\{ & \cos \phi_{p}\left[\cos \left(\theta_{x}-\xi_{x e}\right)\left(1+\beta \sin \xi_{y} \sin \xi_{y e}\right)\right. \\
& \left.+\beta \cos \xi_{y} \cos \xi_{y e}\right]-\sin \phi_{p} \sin \left(\theta_{x}-\xi_{x e}\right)\left(\sin \xi_{y}\right. \\
& \left.\left.+\beta \sin \xi_{y e}\right)\right\}, \\
\alpha_{y e}^{\prime}=\frac{1}{g^{\prime}}\{ & \cos \phi_{p} \sin \left(\theta_{x}-\xi_{x e}\right)\left(\sin \xi_{y e}+\beta \sin \xi_{y}\right) \\
& +\sin \phi_{p}\left[\cos \left(\theta_{x}-\xi_{x e}\right)\left(\beta+\sin \xi_{y} \sin \xi_{y e}\right)\right. \\
& \left.\left.+\cos \xi_{y} \cos \xi_{y e}\right]\right\}
\end{aligned}
$$

and

$$
\begin{aligned}
\alpha_{z e}^{\prime}=\frac{-1}{\gamma g^{\prime}}\{ & \cos \phi_{p} \sin \left(\theta_{x}-\xi_{x e}\right) \cos \xi_{y e} \\
& +\sin \phi_{p}\left[\sin \xi_{y} \cos \xi_{y e} \sin \left(\theta_{x}-\xi_{x e}\right)\right. \\
& \left.\left.-\cos \xi_{y} \sin \xi_{y e}\right]\right\} .
\end{aligned}
$$

Equations (20a)-(20c) express the normalized components of the polarization vector in the electron beam rest frame in terms of the laser and electron direction, the electron beam energy, and laser polarization. It is interesting to note that $\alpha_{z}^{\prime}$ approaches zero as $\gamma$ becomes very large. Thus, for $\gamma \gg 1$, the photon will be incident in the $-z_{e}^{\prime}$ direction, irrespective of the incident angle in the lab frame. It is also worth noting that Eqs. (20a)-(20c) can be simplified significantly in the plane-wave approximation $\left(\xi_{x}=\xi_{y}=0\right)$, resulting in

$$
\begin{gathered}
\alpha_{x e}^{\prime} \approx \frac{1}{g^{\prime}}\left\{\cos \phi_{p}\left[\cos \left(\theta_{x 0}-\xi_{x e}\right)+\beta \cos \xi_{y e}\right]\right. \\
\left.-\beta \sin \phi_{p} \sin \xi_{y e} \sin \left(\theta_{x 0}-\xi_{x e}\right)\right\}, \\
\alpha_{y e}^{\prime} \approx \frac{1}{g^{\prime}}\left\{\cos \phi_{p} \sin \left(\theta_{x 0}-\xi_{x e}\right) \sin \xi_{y e}\right. \\
\left.+\sin \phi_{p}\left[\beta \cos \left(\theta_{x 0}-\xi_{x e}\right)+\cos \xi_{y e}\right]\right\},
\end{gathered}
$$


and

$$
\alpha_{z e}^{\prime} \approx \frac{-1}{\gamma g^{\prime}}\left[\cos \phi_{p} \sin \left(\theta_{x 0}-\xi_{x e}\right) \cos \xi_{y e}-\sin \phi_{p} \sin \xi_{y e}\right]
$$

\section{B. Transformation of incident and scattered photon wave vectors}

Equations (20a)-(20c) and (21a)-(21c) in conjunction with Eq. (9) express the differential Thomson cross section in the electron rest frame in terms of the interaction geometry in the lab frame. The next step is to transform this expression to the electron lab frame $\left(x_{e}, y_{e}, z_{e}\right)$. To accomplish this, we consider the transformation of the incident wave vector $\left(\omega_{0} / c, \mathbf{k}_{0}\right)$ from the lab frame to the rest frame:

$$
\begin{aligned}
& \frac{\omega_{0}^{\prime}}{c}=\gamma\left(\frac{\omega_{0}}{c}-\boldsymbol{\beta} \cdot \mathbf{k}_{0}\right), \\
& k_{0 \|}^{\prime}=\gamma\left(k_{0 \|}-\beta \frac{\omega_{0}}{c}\right),
\end{aligned}
$$

and

$$
k_{0 \perp}^{\prime}=k_{0 \perp},
$$

where $\boldsymbol{\beta}$ is the electron velocity normalized to the speed of light. The lab reference frame is once again defined with respect to the electron beam direction [see Fig. 2(b)], such that Eq. (22) can be rewritten as

$$
\frac{\omega_{0}^{\prime}}{\omega_{0}}=g^{\prime}\left(\theta_{x}, \xi_{y}, \xi_{x e}, \xi_{y e}\right)
$$

where $g^{\prime}$ is the quantity previously defined in Eq. (18).

Within the rest frame, the scattered photon direction will be defined by the wave vector $\mathbf{k}_{s}^{\prime}=$ $\left(\omega_{s}^{\prime} / c\right)\left(\sin \theta_{e}^{\prime} \cos \phi_{e}^{\prime} \hat{x}_{e}^{\prime}+\sin \theta_{e}^{\prime} \sin \phi_{e}^{\prime} \hat{y}_{e}^{\prime}+\cos \theta_{e}^{\prime} \hat{z}_{e}^{\prime}\right)$, where $\theta_{e}^{\prime}$ and $\phi_{e}^{\prime}$ specify the scattered photon direction about the positive $z_{e}^{\prime}$ axis (see Fig. 1). Since only the Thomson limit is being considered, the scattered photon frequency is taken to be equal to the incident frequency $\omega_{0}^{\prime}$. The scattered photon energy in the rest frame, $\omega_{s}^{\prime}$, is expressed in terms of the photon energy in the lab frame, $\omega_{s}$, by once again using the Lorentz transformation from the lab frame to the rest frame:

$$
\frac{\omega_{s}^{\prime}}{c}=\gamma\left(\frac{\omega_{s}}{c}-\boldsymbol{\beta} \cdot \mathbf{k}_{s}\right)=\frac{\omega_{s}}{c} \gamma\left(1-\beta \cos \theta_{e}\right),
$$

where $\theta_{e}$ is the angle of the scattered photon with respect to the $z_{e}$ axis in the electron lab frame. Applying the Thomson limit approximation and inserting Eq. (25) into Eq. (26), leads to

$$
\begin{aligned}
\frac{\omega_{s}}{\omega_{0}} & =\frac{g^{\prime}\left(\theta_{x}, \xi_{y}, \xi_{x e}, \xi_{y e}\right)}{\gamma\left[1-\beta \cos \left(\theta_{e}\right)\right]} \equiv g\left(\theta_{x}, \xi_{y}, \xi_{x e}, \xi_{y e}, \theta_{e}\right) \\
& \approx \frac{2 \gamma g^{\prime}\left(\theta_{x}, \xi_{y}, \xi_{x e}, \xi_{y e}\right)}{1+\gamma^{2} \theta_{e}^{2}} .
\end{aligned}
$$

For a head-on collision $\left(\theta_{x}=\xi_{y}=\xi_{x e}=\xi_{y e}=0\right)$, it is seen that the energy of the scattered photon is maximally up-shifted by a factor of $4 \gamma^{2}$, while for a side-on collision, the up-shift is $2 \gamma^{2}$. It is also seen that the scattered photon energy distribution is the familiar Lorentzian function of observation angle $\theta_{e}$ with a FWHM equal to $1 / \gamma$.

The scattered photon direction in the rest frame can be expressed in terms of lab angles by transforming $\mathbf{k}_{s}$ back into the rest frame. Using the analogs of Eqs. (23) and (24) we obtain

$$
\begin{gathered}
\cos \theta_{e}^{\prime}=\frac{\omega_{s}}{\omega_{s}^{\prime}} \gamma\left(\cos \theta_{e}-\beta\right)=\frac{\cos \theta_{e}-\beta}{1-\beta \cos \theta_{e}}, \\
\sin \theta_{e}^{\prime} \cos \phi_{e}^{\prime}=\frac{\sin \theta_{e} \cos \phi_{e}}{\gamma\left(1-\beta \cos \theta_{e}\right)},
\end{gathered}
$$

and

$$
\sin \theta_{e}^{\prime} \sin \phi_{e}^{\prime}=\frac{\sin \theta_{e} \sin \phi_{e}}{\gamma\left(1-\beta \cos \theta_{e}\right)},
$$

where $\phi_{e}$ is the azimuthal angle about $z_{e}$ in the electron lab frame. Equations (28)-(30) can be inserted into Eq. (9) to obtain the rest frame differential cross section in lab frame coordinates. To get an expression for the lab frame differential cross section, we have

$$
\begin{aligned}
\frac{d \sigma}{d \Omega} & =\frac{d \sigma}{d \Omega^{\prime}} \frac{d \Omega^{\prime}}{d \Omega}=\frac{d \sigma}{d \Omega^{\prime}} \frac{d\left(\cos \theta_{e}^{\prime}\right)}{d \cos \theta_{e}} \\
& =\frac{d \sigma}{d \Omega^{\prime}}\left(\frac{1-\beta^{2}}{\left[1-\beta \cos \left(\theta_{e}\right)\right]^{2}}\right) .
\end{aligned}
$$

Here, $d \Omega=\sin (\theta) d \theta d \phi=-d \cos (\theta) d \phi$, and we have used the fact that $d \phi=d \phi^{\prime}$. This expression, along with Eqs. (9) and (28)-(30), lead to the expression for the lab frame differential cross section, given by

$$
\begin{aligned}
\frac{d \sigma}{d \Omega}\left(\theta_{e}, \phi_{e}\right) \frac{\left[1-\beta \cos \left(\theta_{e}\right)\right]^{2}}{r_{0}^{2}\left(1-\beta^{2}\right)}= & \alpha_{x}^{\prime 2}\left(1-\frac{\cos ^{2}\left(\phi_{e}\right) \sin ^{2}\left(\theta_{e}\right)}{\gamma^{2}\left[1-\beta \cos \left(\theta_{e}\right)\right]^{2}}\right)+\alpha_{y}^{\prime 2}\left(1-\frac{\sin ^{2}\left(\phi_{e}\right) \sin ^{2}\left(\theta_{e}\right)}{\gamma^{2}\left[1-\beta \cos \left(\theta_{e}\right)\right]^{2}}\right)+\alpha_{z}^{\prime 2}\left[1-\left(\frac{\cos \left(\theta_{e}\right)-\beta}{1-\beta \cos \left(\theta_{e}\right)}\right)^{2}\right] \\
& -2 \alpha_{x}^{\prime} \alpha_{y}^{\prime} \frac{\cos \left(\phi_{e}\right) \sin \left(\phi_{e}\right) \sin ^{2}\left(\theta_{e}\right)}{\gamma^{2}\left[1-\beta \cos \left(\theta_{e}\right)\right]^{2}}-2 \alpha_{x}^{\prime} \alpha_{z}^{\prime} \frac{\left.\cos \left(\theta_{e}\right)-\beta\right] \cos \left(\phi_{e}\right) \sin \left(\theta_{e}\right)}{\gamma\left[1-\beta \cos \left(\theta_{e}\right)\right]^{2}} \\
& -2 \alpha_{y}^{\prime} \alpha_{z}^{\prime} \frac{\left[\cos \left(\theta_{e}\right)-\beta\right] \sin \left(\phi_{e}\right) \sin \left(\theta_{e}\right)}{\gamma\left[1-\beta \cos \left(\theta_{e}\right)\right]^{2}}
\end{aligned}
$$


Note that while the cross section has been expressed as a function of only the observation angles $\theta_{e}$ and $\phi_{e}$, the dependence on the initial interaction geometry $\left(\theta_{x}, \xi_{y}, \xi_{x e}, \xi_{y e}\right)$ is implicit in the components for the polarization vector [Eqs. (20a)-(20c)]. Finally, we wish to express this cross section in the stationary lab frame coordinate system $(x, y, z)$. To accomplish this, we consider the transformation from the electron lab frame $\left(x_{e}\right.$, $\left.y_{e}, z_{e}\right)$ to the stationary lab frame $(x, y, z)$. Noting that $r_{e}=r$, and

$$
x=r \cos \phi \sin \theta, \quad y=r \sin \phi \sin \theta, \quad z=r \cos \theta,
$$

and performing the transformation

$$
\mathbf{r}_{e}=\left(\mathbb{R}_{e y} \mathbb{R}_{e x}\right) \mathbf{r}
$$

we have the following expressions for the relevant trigonometric terms in the rotated electron frame used in Eq. (32) in terms of the stationary laboratory frame coordinates $(\theta, \phi)$ and the relative electron direction $\left(\xi_{x e}, \xi_{y e}\right)$ :

$$
\cos \phi_{e} \sin \theta_{e}=\cos \phi \sin \theta \cos \xi_{x e}-\cos \theta \sin \xi_{x e},
$$

$\sin \phi_{e} \sin \theta_{e}=\sin \phi \sin \theta \cos \xi_{y e}-\cos \phi \sin \theta \sin \xi_{y e} \cos \xi_{x e}$

$$
-\cos \theta \cos \xi_{x e} \sin \xi_{y e}
$$

and

$$
\begin{aligned}
\cos \theta_{e}= & \cos \theta \cos \xi_{x e} \cos \xi_{y e}+\sin \phi \sin \theta \sin \xi_{y e} \\
& +\cos \phi \sin \theta \sin \xi_{x e} \cos \xi_{y e} .
\end{aligned}
$$

Equation (32) combined with Eqs. (20a)-(20c) and (35)(37) completely describe the probability distribution of scattered photons in the lab frame for the collision of a relativistic electron with a photon at arbitrary incident angle and polarization.

\section{GENERAL PROPERTIES OF THOMSON SCATTERING}

The cross section described by Eq. (32) can be used to present a brief review of the basic properties of Thomson scattering. We consider the case of a single electron $\left(\xi_{x e}=\right.$ $\left.\xi_{y e}=0\right)$ colliding with a plane-wave $\left(\xi_{x}=\xi_{y}=0\right)$ incident at an angle $\theta_{0}$ with respect to the electron direction. As discussed in Sec. I, the rate of scattered photons per unit solid angle will be proportional to $d \sigma / d \Omega$ such that

$$
\frac{d N_{s}}{d \Omega d t}(\theta, \phi)=F_{\omega_{0}}(t) \frac{d \sigma}{d \Omega}(\theta, \phi),
$$

where $F_{\omega_{0}}$ represents the incident photon flux and is given by [see Eq. (4)]

$$
F_{\omega_{0}}(t)=c\left(1-\frac{c}{\omega_{0}} \boldsymbol{\beta}_{e} \cdot \mathbf{k}_{0}\right) n_{\gamma}\left[\mathbf{r}_{e}(t), t\right] .
$$

Figures 3 and 4 illustrate the characteristics of the differ-

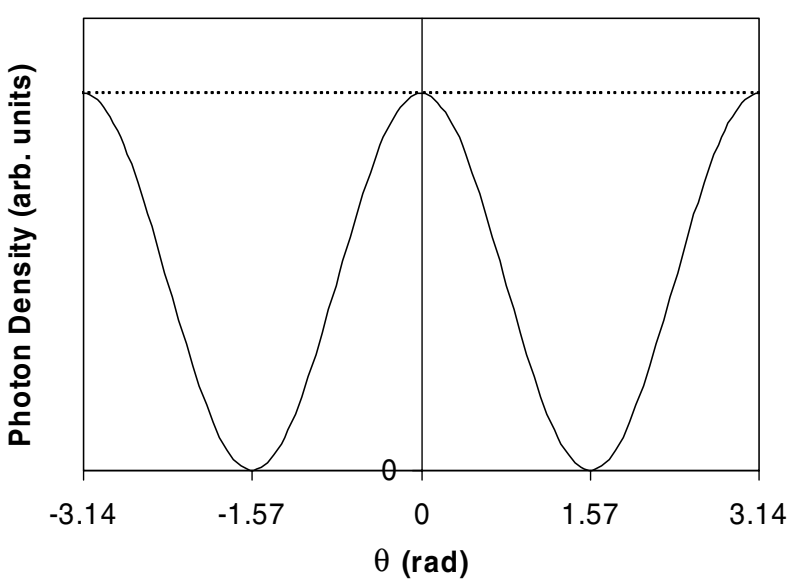

FIG. 3. Scattered photon density vs scattering angle $(\theta)$ from the $z$ axis in the $x-z$ plane (solid line) and the $y-z$ plane (dotted line) for the case of an electron at rest and an $x$ polarized photon incident along the negative $z$ axis.

ential cross section for the case of a head-on collision $\left(\theta_{0}=0\right)$, with polarization in the $x$ direction. To illustrate the differences between the cross section for a nonrelativistic and relativistic electron, two cases $(\gamma=1$ and $\gamma=2$ ) are illustrated. For the case of the nonrelativistic electron, the scattered photon density follows that of a dipole radiation pattern, with the density being uniform in the plane perpendicular to the incident polarization vector ( $y-z$ plane), and with the density falling to zero in the direction of the polarization vector in the $x-z$ plane. For the relativistic case, the scattered photon density is collimated in the direction of the electron beam in a cone of angular FWHM roughly equal to $1 / \gamma$. However, the probability distribution is broader in the plane perpendicular to the plane of polarization than in the plane

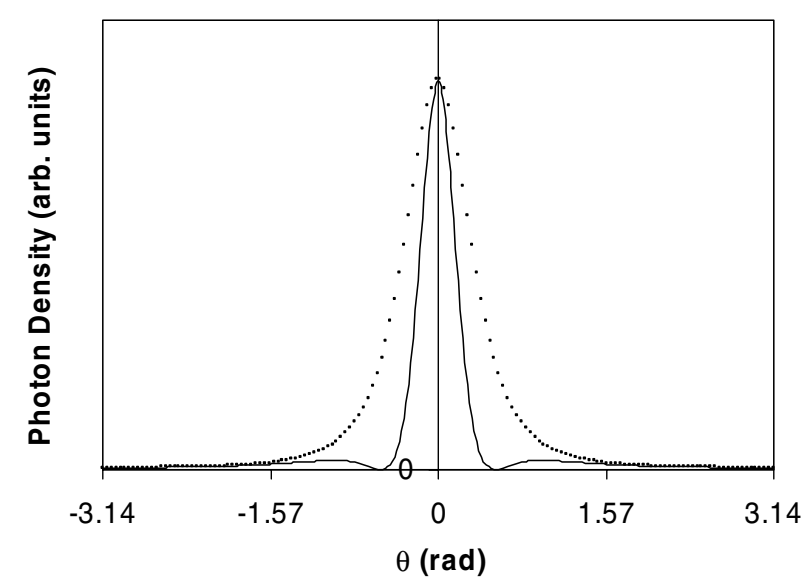

FIG. 4. Scattered photon density vs scattering angle $(\theta)$ from the $z$ axis in the $x-z$ plane (solid line) and the $y-z$ plane (dotted line) for the case of a relativistic electron $(\gamma=2)$ traveling along the $z$ axis and an $x$ polarized photon incident along the negative $z$ axis. 
parallel to it, with the intensity in the parallel plane having both a central strong intensity peak on axis, corresponding to the scattered photons from the forward direction in the rest frame, and the much smaller sidelobe intensity peaks corresponding to the photons in the backward direction in the rest frame. Note that in Figs. 3 and $4, \theta$ is plotted from $-\pi$ to $\pi$, where negative values of $\theta$ correspond to a $180^{\circ}$ rotation of the $\phi$ coordinate.

While Figs. 3 and 4 provide detailed information on the photon density, it is often desirable to express the scattered $x$-ray beam in terms of the energy density, since this is often what is directly measured. To this end, the scattered x-ray intensity distribution can be obtained by simply multiplying Eq. (38) by the energy of the scattered photon, resulting in

$$
\frac{d U_{s}}{d \Omega d t}(\theta, \phi, t)=\hbar \omega_{0} g(\theta) F_{\omega 0}(t) \frac{d \sigma}{d \Omega}(\theta, \phi) .
$$

The scattered energy density is plotted in Fig. 5 for the case of a relativistic electron with $\gamma=2$. The intensity profile is somewhat narrower than the photon probability distribution due to the fact that lower energy photons correspond to the photons with the larger divergence angles from the electron direction. It is also worth noting that the outside lobes evident in the $x-z$ plane of the photon probability distribution are less significant in the energy intensity distribution.

For the case of an off-axis photon incident angle (e.g., side-on collision), the differences in the intensity/probability profiles are minor for a sufficiently relativistic electron. This fact is obvious when considering that in the rest frame of the electron, the incident angle of the incoming photon scales roughly as $1 / \gamma$, closely simulating a head-on collision for a sufficiently relativistic beam. However, if the photon is polarized in the plane of in-

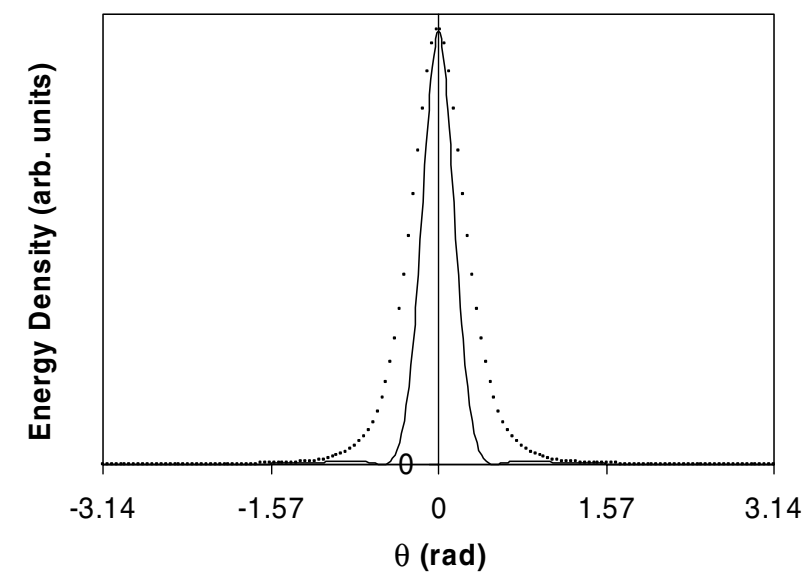

FIG. 5. Scattered energy density vs scattering angle $(\theta)$ from the $z$ axis in the $x-z$ plane (solid line) and the $y-z$ plane (dotted line) for the case of a relativistic electron $(\gamma=2)$ traveling in along the $z$ axis and an $x$ polarized photon incident in the negative $z$ direction. cidence, there will be a slight offset in the photon/intensity distribution in this plane. This is seen in Fig. 6. On the other hand, it can be seen from Eq. (32) that if the photon polarization is perpendicular to the plane of incidence of the collision (i.e., $\alpha_{x}^{\prime}=\alpha_{z}^{\prime}=0$ ), then the photon probability distribution is independent of incidence angle for all electron beam energies.

By utilizing the approximate independence of the scattered photon probability distribution on the incident photon polarization in the high $\gamma$ limit, a greatly simplified expression for the cross section can be obtained. If we take the case $\alpha_{x}=\alpha_{z}=0$, and we once again assume $\xi_{x e}=\xi_{y e}=\xi_{x}=\xi_{y}=0$, then for $\gamma \gg 1$, Eq. (25) becomes

$$
\frac{d \sigma}{d \Omega} \approx r_{0}^{2} \frac{4 \gamma^{2}}{\left(1+\theta^{2} \gamma^{2}\right)^{2}}\left[1-\frac{4\left(\theta^{2} \gamma^{2}\right)}{\left(1+\theta^{2} \gamma^{2}\right)^{2}} \sin ^{2}(\phi)\right],
$$

while the scattered intensity distribution becomes

$$
\begin{aligned}
\frac{d U_{s}}{d \Omega d t} \approx & \hbar \omega_{0} F_{\omega 0}(t) \frac{8 \gamma^{4}\left[1+\beta \cos \left(\theta_{0}\right)\right]}{\left(1+\theta^{2} \gamma^{2}\right)^{3}} \\
& \times\left[1-\frac{4\left(\theta^{2} \gamma^{2}\right)}{\left(1+\theta^{2} \gamma^{2}\right)^{2}} \sin ^{2}(\phi)\right] .
\end{aligned}
$$

Equations (41) and (42) will be approximately correct for an arbitrary linear laser polarization provided the coordinate axis is defined such that $\phi=\pi / 2$ corresponds to the plane of polarization, and $\gamma \gg 1$. In this case, the scattered intensity profile in the plane perpendicular to the laser polarization is described by a cubed Lorentzian with intensity FWHM of $1 / \gamma$. In the plane parallel to the laser polarization, the intensity profile is described by a superposition of a cubed Lorentzian and a Lorentzian to the fifth power, where the scattered intensity will go to zero at $\theta=1 / \gamma$, and the FWHM will occur at $\theta \approx$ $0.635 / \gamma$.

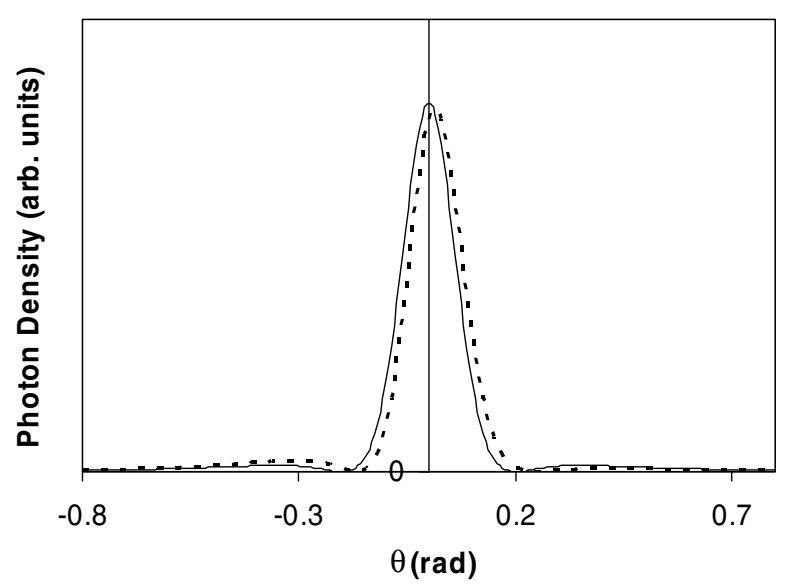

FIG. 6. Scattered photon density from a relativistic electron $(\gamma=5)$ in the $x-z$ plane produced by an $x$ polarized photon for the case of head-on interaction (solid line) and a side-on interaction (dotted line). 


\section{SPECTRAL BROADENING FROM THREE- DIMENSIONAL EFFECTS}

The strict correlation between scattering angle and wavelength implies that, in principle, the spectrum produced from Thomson backscattering can be very narrow at a given observation angle. However, the threedimensional aspects of the interaction geometry will, in reality, lead to significant broadening of the spectrum at a given observation position. In Sec. IVA, the spectral broadening due to the finite bandwidth in the laser pulse will be introduced into the theory. In Sec. IV B, the effects from the perpendicular $k$ vector components within the laser focus will be considered. Finally, in Sec. IVC, the spectral broadening due to the energy spread and transverse emittance in the electron beam will be considered.

\section{A. Laser bandwidth}

Thomson scattering is often performed with a short ( $\mathrm{ps}$ to fs) laser pulse. Consequently, the amount of bandwidth in the incident laser pulse can become a significant quantity. For the case of a Fourier-transform limited laser pulse, the $1 / e^{2}$ width of the photon frequency distribution will be given by the Fourier limit relation

$$
\Delta \omega_{0} \Delta t_{0}=2
$$

where $\Delta t_{0}$ and $\Delta \omega_{0}$ are the $1 / e^{2}$ temporal and spectral widths, and a Gaussian distribution has been assumed. To generalize to the case of chirped pulses that are not Fourier-transform limited, both the center wavelength of the laser pulse and the spectral width can be expressed as a function of the longitudinal position within the laser pulse, $\zeta(t)$, with the time-dependent spectral density distribution given by

$$
n_{\omega}[\omega, \Delta \omega, \zeta(t)]=\sqrt{\frac{2}{\pi}} \frac{1}{\Delta \omega_{0}(\zeta)} \exp \left(-\frac{2\left[\omega-\omega_{0}(\zeta)\right]^{2}}{\Delta \omega_{0}^{2}(\zeta)}\right),
$$

where $\zeta(t)=z_{L}+c t$, where $z_{L}$ is measured along the rotated laser frame corresponding to the average $k$ vector of the laser pulse, and $n_{\omega}$ is the probability distribution for a photon within the laser beam having a wavelength corresponding to $\omega$. The time-dependent photon spectral density flux for the scattered $x$ rays [Eq. (5)], now becomes

$$
\frac{d N_{s}}{d \Omega d \omega_{s} d t}=\int F_{\omega 0}(t) \frac{d \sigma}{d \Omega}(\theta, \phi) n_{\omega}\left(\omega_{i}, \Delta \omega_{0}\right) \delta\left[\omega_{s}-\omega_{i} g(\theta)\right] d \omega_{i}
$$

This expression can be integrated using the relation

$$
\delta\left[\omega_{s}-\omega_{i} g(\theta)\right]=\frac{\delta\left[\omega_{i}-\omega_{s} / g(\theta)\right]}{|g(\theta)|},
$$

resulting in

$$
\frac{d N_{s}}{d \Omega d \omega_{s} d t}=F_{\omega 0}(t) \sqrt{\frac{2}{\pi}} \frac{\frac{d \sigma}{d \Omega}(\theta, \phi)}{\Delta \omega_{0}|g(\theta)|} \exp \left(-\frac{2\left[\frac{\omega_{s}}{g(\theta)}-\omega_{0}(\zeta)\right]^{2}}{\Delta \omega_{0}^{2}(\zeta)}\right) .
$$

Similarly, the time-dependent energy spectral density flux is given by

$$
\left.\frac{d U_{s}}{d \Omega d \omega_{s} d t}=\hbar \omega_{0}(\zeta) F_{\omega 0}(t) \sqrt{\frac{2}{\pi} \frac{d \sigma}{d \Omega}(\theta, \phi)} \frac{2\left[\frac{\omega_{s}}{g(\theta)}-\omega_{0}(\zeta)\right]^{2}}{\Delta \omega_{0}^{2}(\zeta)}\right)
$$

Thus, the percent bandwidth of the scattered $\mathrm{x}$ rays due to the above mechanism is equal to the percent bandwidth of the incident laser pulse, such that

$$
\frac{\Delta \omega_{s}}{\omega_{s}} \approx \frac{\Delta \omega_{0}}{\omega_{0}}
$$

\section{B. Laser focus effects}

Equations (47) and (48) contain the spectral and temporal information for the Thomson scattered radiation from a single electron colliding with collimated photons (or an electromagnetic wave in the plane-wave approximation). To include nonplane wave effects in the calculation, we consider the distribution of perpendicular $k$-vector components within the laser pulse focus as representing the spread in the direction of the incoming photons, while representing the laser focus within the paraxial approximation $[26,27]$.

We begin by considering the spatial distribution of photons near the laser beam focus. In so doing, a central laser frame $\left(x_{L}, y_{L}, z_{L}\right)$ is defined such that the $z_{L}$ axis corresponds to the direction opposite the direction of the average wave vector in the laser pulse, $\overline{\mathbf{k}}_{0}$. Recalling that the laser pulse is assumed to be incident at an angle $\theta_{0}$ in the $x-z$ plane, we have

$$
\overline{\mathbf{k}}_{0}=-k_{0} \hat{z}_{L}=-k_{0}\left[\sin \left(\theta_{0}\right) \hat{x}+\cos \left(\theta_{0}\right) \hat{z}\right] .
$$

The expression for the photon density in the paraxial 
approximation is given by

$$
\begin{aligned}
n_{\gamma}(\mathbf{r}, t)= & \frac{N_{\gamma} \exp \left(\frac{-2\left(c t+z_{L}\right)^{2}}{c^{2} \Delta t^{2}}\right)}{\left(\frac{\pi}{2}\right)^{3 / 2} c \Delta t w_{x 0} w_{y 0} \sqrt{1+z_{L}^{2} / z_{R x}^{2}} \sqrt{1+z_{L}^{2} / z_{R y}^{2}}} \\
& \times \exp \left(\frac{-2 x_{L}^{2}}{w_{x 0}\left(1+z_{L}^{2} / z_{R x}^{2}\right)}\right) \exp \left(\frac{-2 y_{L}^{2}}{w_{x 0}\left(1+z_{L}^{2} / z_{R y}^{2}\right)}\right),
\end{aligned}
$$

where $N_{\gamma}$ represents the total number of photons in the pulse, $z_{R x}$ and $z_{R y}$ represent the Rayleigh range of the laser focus in the $x$ and $y$ dimensions, respectively, and $n_{\gamma}$ is in units of photons/(unit length) ${ }^{3}$. In Eq. (51), it is assumed that the laser focus occurs at $z_{L}=0$. To convert the expression from the laser frame to the unrotated lab frame, we simply replace $x_{L}, y_{L}$, and $z_{L}$ with the following relations:

$$
x_{L}=x \cos \left(\theta_{0}\right)-z \sin \left(\theta_{0}\right), \quad y_{L}=y,
$$

and

$$
z_{L}=z \cos \left(\theta_{0}\right)+x \sin \left(\theta_{0}\right)
$$

The Rayleigh range of the laser focus is given by the expression [26]

$$
z_{\mathrm{R}}=\pi \frac{w_{0}^{2}}{M^{2} \lambda_{0}}
$$

where $w_{0}$ is the $1 / e^{2}$ intensity radius in $x$ or $y, \lambda_{0}$ is the average wavelength of the laser pulse, and $M^{2}>1$ deter- mines how close the focus is to being diffraction limited. Using the analogy of the Rayleigh range to the beta function of a particle beam focus, it is possible to express an effective $1 / e^{2}$ "emittance" of the laser beam as [26]

$$
\varepsilon_{L}=\frac{M^{2} \lambda_{0}}{\pi}=w_{0} w_{0}^{\prime},
$$

where $w_{0}^{\prime} \equiv \Delta \xi$ is the $1 / e^{2}$ width of the divergence of the laser beam, and, consequently, the $1 / e^{2}$ width in the distribution of photon directions at the focus. For a sufficiently soft focus, $\Delta \xi_{x}=\left(\Delta k_{x} / k_{0}\right)$ and $\Delta \xi_{y}=\left(\Delta k_{y} / k_{0}\right)$, and

$$
f_{k \perp}\left(\xi_{x}, \xi_{y}\right)=\frac{2}{\pi \Delta \xi_{x} \Delta \xi_{y}} \exp \left(\frac{-2 \xi_{x}^{2}}{\Delta \xi_{x}^{2}}\right) \exp \left(\frac{-2 \xi_{y}^{2}}{\Delta \xi_{y}^{2}}\right)
$$

where $\Delta \xi_{x}=\left(\varepsilon_{L} / w_{0 x}\right)$. The distribution is normalized such that

$$
\iint f_{k \perp}\left(\xi_{x}, \xi_{y}\right) d \xi_{x} d \xi_{y}=1
$$

It should be noted that for a coherent beam, the distribution of $k_{\perp}$ represents the spread in the direction of each photon in the beam, rather than simply the collective spread of all the photons. This differs from the physical interpretation of the electron beam divergence (next section), in which it is relatively accurate to assume that each electron in the bunch has a well-defined direction in comparison to the ensemble average of the electron beam direction as a whole.

Integrating over all incident $k_{\perp}$, the expression for the photon spectral density flux becomes

$$
\frac{d N_{s}}{d \Omega d \omega_{s} d t}=F_{\omega 0}(t) \cdot \iint f_{k \perp}\left(\xi_{x}, \xi_{y}\right) \sqrt{\frac{2}{\pi}} \frac{\frac{d \sigma}{d \Omega}\left(\xi_{x}, \xi_{y}, \theta, \phi\right)}{\Delta \omega_{0}(\zeta)\left|g\left(\xi_{x}, \xi_{y}, \theta\right)\right|} \exp \left\{-\frac{2\left[\frac{\omega_{s}}{g\left(\xi_{x}, \xi_{y}, \theta\right)}-\omega_{0}(\zeta)\right]^{2}}{\Delta \omega_{0}^{2}(\zeta)}\right\} d \xi_{x} d \xi_{y} .
$$

Note that for the case of a plane wave, $\Delta \xi_{x}=\Delta \xi_{y}=0$, and Eq. (57) reduces to Eq. (47). The primary effect of the nonzero spread in $k_{\perp}$ is to broaden the spectrum of the scattered $x$ rays at a given observation direction. This is apparent from Eq. (19), which shows the dependence of the up-shift of the photon frequency in the electron beam rest frame on the relative direction between the photon and the electron. For the case of a head-on collision, this broadening will scale quadratically with $k_{\perp}$, making this effect small, and in many cases, negligible compared to the spectral broadening from other causes, such as laser bandwidth (see Fig. 7). In terms of the focal spot size, this broadening can be expressed as

$$
\left.\frac{\Delta \omega_{s}}{\omega_{s}}\right|_{0^{0}} \approx \frac{\Delta \xi_{x}^{2}}{4}=\frac{1}{4}\left(\frac{\lambda_{0}}{\pi w_{0}}\right)^{2}
$$

for a head-on collision, where $w_{0}$ is the $1 / e^{2}$ intensity radius, and $M^{2}$ was assumed to be equal to unity. On the other hand, for a side-on collision, the scattered $\mathrm{x}$-ray energy is much more sensitive to $k_{\perp}$, as is illustrated in
Fig. 8. For this case

$$
\left.\frac{\Delta \omega_{s}}{\omega_{s}}\right|_{90^{\circ}} \approx \Delta \xi_{x}=\frac{\lambda_{0}}{\pi w_{0}} .
$$

As will be demonstrated later, however, even if the interaction geometry is not head on, the spectral broadening induced by the spread in $k_{\perp}$ will for most cases be insignificant compared to that induced by the finite electron beam emittance. Thus, except for the cases of an extremely strong laser focus or a very low emittance electron beam, a plane-wave approximation is generally adequate for determining the Thomson $\mathrm{x}$-ray spectrum.

\section{Spectral broadening from the electron beam}

Until now, only scattering from a single electron has been considered. In reality, Thomson scattering is performed with a beam containing many electrons. In this treatment, we assume the $\mathrm{x}$ rays are incoherently scattered from the electron bunch. Consequently, the calculated 
total x-ray spectrum will simply be a linear superposition of the spectrum produced by each individual electron. Spectral broadening of the $x$ rays will occur from (i) the spread in energy of the electrons in the bunch, and (ii) the finite divergence of the beam at the interaction.

Since the scattered $x$-ray energy scales as the square of the electron beam energy, the spectral broadening at a given observation angle due to the electron beam energy spread can be expressed as

$$
\frac{\Delta \omega_{s}(\theta)}{\bar{\omega}_{s}(\theta)} \approx 2 \frac{\Delta \gamma}{\bar{\gamma}}
$$

where $\bar{\omega}_{s}$ is the average scattered $\mathrm{x}$-ray energy at the observation angle $\theta$ and $\bar{\gamma}$ is the average relativistic Lorentz factor of the electron beam. For a high quality electron beam, the rms energy spread is typically on the order of $0.1 \%$. Consequently, the effect will most likely be comparable to smaller than the spectral broadening due to the laser pulse bandwidth for collisions with short $(<1 \mathrm{ps})$ laser pulses.

A potentially much more significant contribution to the spectral broadening of the $\mathrm{x}$-ray pulse is the finite electron beam emittance. The small value of the Thomson cross section dictates that both the electron bunch and the laser pulse be focused to a very small spot size $(<100 \mu \mathrm{m})$ to produce a large number of backscattered $\mathrm{x}$ rays. For an electron beam of rms geometric emittance, $\varepsilon_{x}$, the rms divergence of the electrons at the beam waist will be given by

$$
\sigma_{e x^{\prime}}=\frac{\varepsilon_{x}}{\sigma_{e x}} \equiv \frac{\Delta \xi_{x e}}{2}
$$

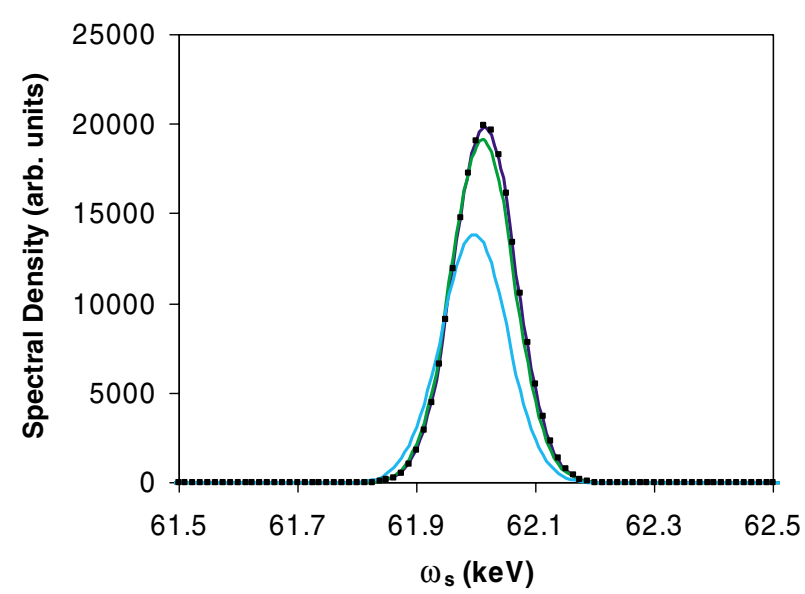

FIG. 7. (Color) On-axis X-ray spectrum produced by a $\gamma=100$ electron colliding head on with an $800 \mathrm{~nm}$ laser pulse with a bandwidth corresponding to a $0.5 \mathrm{ps} 1 / e^{2}$ pulse width for the case of a plane wave (dots), $20 \mu \mathrm{m}$ laser focus (dark blue line), $10 \mu \mathrm{m}$ laser focus (green line), and $5 \mu \mathrm{m}$ laser focus (light blue line). where $\sigma_{e x}$ is the rms spot size in the $x$ dimension at the electron beam focus, and $\Delta \xi_{x e}$ is the $1 / e^{2}$ divergence of the electron beam (assuming a Gaussian distribution). Equation (61) is directly analogous to Eq. (54) describing the spread in $k_{\perp}$ for the laser focus. However, the effect on the scattered $\mathrm{x}$-ray spectrum is quite different. In the relativistic limit, the $\mathrm{x}$ rays are scattered primarily in the direction of the electron, and not that of the incoming photon. Thus, while the spreading due to $\Delta k_{\perp}$ is primarily a result of a shift in the maximum scattered photon energy due to the slight change in the relative incident angle between the electron and the incident wave vector, the primary broadening mechanism from the electron beam divergence results from a shift in the center direction of the scattered $\mathrm{x}$-ray distribution for each electron, resulting in a corresponding spread in $x$-ray energies at any given observation point. From Eq. (27), the on-axis spectral broadening can be estimated for small electron beam divergence angles for the case of head-on interaction geometry by replacing $\theta$ with $\Delta \xi_{x e}^{2}+\Delta \xi_{y e}^{2}$, and expanding to first order in $\gamma^{2}\left(\Delta \xi_{x e}^{2}+\Delta \xi_{y e}^{2}\right)$ to obtain

$$
\left.\frac{\Delta \omega_{s}}{\omega_{s}}\right|_{\theta_{x 0}=0, \theta=0} \approx \frac{\gamma^{2}\left(\Delta \xi_{x e}^{2}+\Delta \xi_{y e}^{2}\right)}{2} .
$$

Including the effects of the laser bandwidth and divergence, as well as the electron beam energy spread, we have for the on-axis spectrum for a head-on collision:

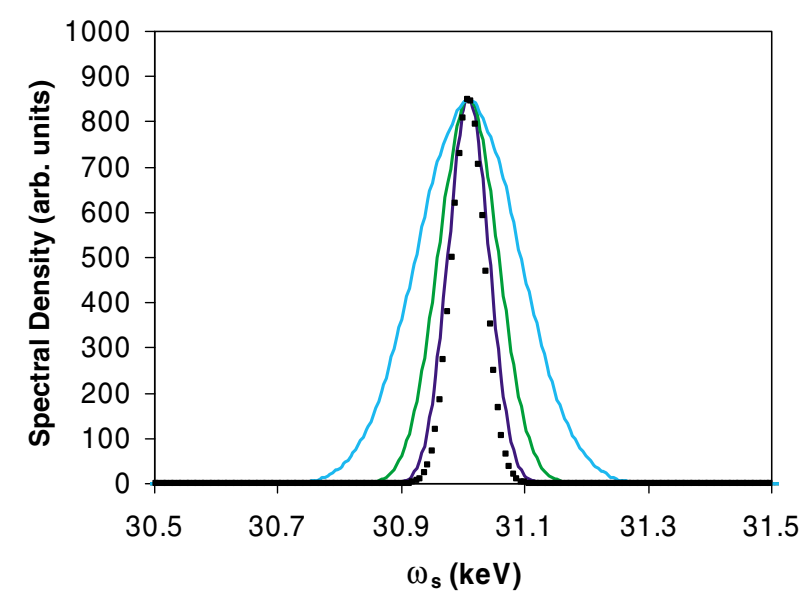

FIG. 8. (Color) On-axis X-ray spectrum produced by a $\gamma=100$ electron colliding side on with an $800 \mathrm{~nm}$ laser pulse with a bandwidth corresponding to a $0.5 \mathrm{ps} 1 / e^{2}$ pulse width for the case of a plane wave (dots), $200 \mu \mathrm{m}$ laser focus (dark blue line), $100 \mu \mathrm{m}$ laser focus (green line), and $50 \mu \mathrm{m}$ laser focus (light blue line). 


$$
\left.\frac{\Delta \omega_{s}}{\omega_{s}}\right|_{\theta_{x 0}=0, \theta=0} \approx \sqrt{\frac{\gamma^{4}\left(\Delta \xi_{x e}^{2}+\Delta \xi_{y e}^{2}\right)^{2}}{4}+4 \frac{\Delta \gamma^{2}}{\gamma^{2}}+\left(\frac{\Delta \xi_{x L}^{2}+\Delta \xi_{y L}^{2}+\Delta \xi_{x e}^{2}+\Delta \xi_{y e}^{2}}{8}\right)^{2}+\frac{\Delta \omega_{0}^{2}}{\omega_{0}^{2}}}
$$

More rigorously, the effect of the electron beam focus on the differential cross section, and hence, the scattered photon spectral density flux, can be obtained by integrating over all the electrons in a given 6D distribution, $f_{e}\left[\xi_{x e}, \xi_{y e}, \gamma_{e}, \mathbf{r}_{e}(0)\right]$. The photon spectral density flux now becomes

$$
\frac{d N_{T}\left(\theta, \phi, \omega_{s}, t\right)}{d \Omega d \omega_{s} d t}=N_{e} \int f_{e}\left[\xi_{x e}, \xi_{y e}, \gamma_{e}, \mathbf{r}_{e}(t)\right] \frac{d N_{s e}\left(\xi_{x e}, \xi_{y e}, \theta, \phi, \omega_{s}, t\right)}{d \Omega d \omega_{s} d t} d \xi_{x e} d \xi_{y e} d \gamma_{e} d^{3} r
$$

where $N_{s e}$ represents the photons scattered by a single electron [Eq. (57)], $N_{e}$ is the total number of electrons in the bunch, and $f_{e}\left[\xi_{x e}, \xi_{y e}, \gamma_{e}, \mathbf{r}_{e}(0)\right]$ is the $6 \mathrm{D}$ phase space probability distribution.

The time and frequency-domain code presented in this paper represents $f_{e}\left[\xi_{x e}, \xi_{y e}, \gamma_{e}, \mathbf{r}_{e}(0)\right]$ with a group of macroparticles rather than an analytic expression. This allows more general electron distributions to be considered, as well as enabling the simulation of electron beams produced from particle dynamics codes. The total number of photons per unit solid angle per unit photon energy for an electron bunch colliding with a laser beam can then be calculated by summing Eq. (57) over all the electrons in the bunch, such that

$$
\frac{d N_{T}\left(\theta, \phi, \omega_{s}, t\right)}{d \Omega d \omega_{s} d t}=\sum_{e} \frac{q_{e}}{e} \frac{d N_{s e}\left(\xi_{x e}, \xi_{y e}, \theta, \phi, \omega_{s}, t\right)}{d \Omega d \omega_{s} d t},
$$

where $q_{e}$ is the charge represented by the macroparticle, and the subscript $e$ has been employed to denote each macroparticle in the calculation.

\section{TIME AND FREQUENCY-DOMAIN CODE DESCRIPTION}

Equation (65) represents the basic algorithm of the 3D time and frequency-domain code. In its most general form, the program calculates the number of photons scattered into a given solid angle and range of $\omega_{s}$ at each time step by summing over all incident $k_{\perp}$ within the laser pulse. This will be performed for all the electrons in the bunch, which are represented by a series of macroparticles with charge equal to $q_{e}$. The background motion of the electron through the laser pulse is assumed to be ballistic. The temporal information of the $\mathrm{x}$-ray pulse is determined by calculating the time of flight of the scattered photon to a detector at a specified distance to the interaction at each time step in the simulation. Spatial information of the scattered $\mathrm{x}$-ray pulses is determined by performing this calculation for several different observation directions specified by $\theta$ and $\phi$. To review the assumptions inherent in the 3D time and frequency-domain code, we state that (i) the normalized vector potential of the incident laser pulse, $e A / m c$, is much less than 1, (ii) the incident photon energy in the electron's rest frame is much less than the electron rest mass (i.e., $\hbar \omega_{0}^{\prime} \ll m c^{2}$ ), and (iii) the scattered $\mathrm{x}$-ray wavelength is much shorter than the size of the electron bunch (i.e., incoherent scattering).

There are a few simplifications that can be made under some circumstances to significantly reduce the amount of computation. One is to make the assumption that the incident laser pulse is a plane wave. For this case, no integration of the incident $k$ vectors is performed, and Eq. (57) becomes

$$
\left.\frac{d N_{s e}\left(\xi_{x e}, \xi_{y e}, \theta, \phi, \omega_{s}, t\right)}{d \Omega d \omega_{s} d t}\right|_{k_{\perp}=0}=F_{\omega 0}(t) \cdot \sqrt{\frac{2}{\pi}} \frac{\frac{d \sigma}{d \Omega}\left(\xi_{x e}, \xi_{y e}, \theta, \phi\right)}{\Delta \omega_{0}(\zeta(t))\left|g\left(\xi_{x e}, \xi_{y e}, \theta\right)\right|} \exp \left\{-\frac{2\left[\frac{\omega_{s}}{g\left(\xi_{x e}, \xi_{y e}, \theta\right)}-\omega_{0}(\zeta(t))\right]^{2}}{\Delta \omega_{0}^{2}(\zeta(t))}\right\}
$$

Another simplification is the assumption that there is no temporal dependence of the average wavelength of the incident laser pulse (i.e., it is bandwidth limited in time). Under this circumstance, rather than calculating everything at each time step, the time integration can be separated out of the calculation of the spectral and angular dependence of the scattered x-ray distribution, resulting in only one required evaluation of the cross section for each scattered frequency and solid angle for each electron in the bunch. In this case, the time integration of Eq. (57) will result in

$$
\frac{d N_{s e}\left(\xi_{x e,} \xi_{y e}, \theta, \phi, \omega_{s}\right)}{d \Omega d \omega_{s}}=\int F_{\omega 0}(t) d t \iint f_{k \perp}\left(\xi_{x}, \xi_{y}\right) \sqrt{\frac{2}{\pi}} \frac{\frac{d \sigma}{d \Omega}\left(\xi_{x e}, \xi_{y e}, \xi_{x}, \xi_{y}, \theta, \phi\right)}{\Delta \omega_{0}\left|g\left(\xi_{x e}, \xi_{y e}, \xi_{x}, \xi_{y}, \theta\right)\right|} \exp \left\{-\frac{2\left[\frac{\omega_{s}}{g\left(\xi_{x e}, \xi_{y e}, \xi_{x}, \xi_{y}, \theta\right)}-\omega_{0}\right]^{2}}{\Delta \omega_{0}^{2}}\right\} d \xi_{x} d \xi_{y}
$$

where the time dependence of the central laser frequency $\omega_{0}$ and the bandwidth $\Delta \omega_{0}$ has been removed. Under this assumption, the temporal information of the scattered $\mathrm{x}$ rays is obtained by assuming all the photons scattered into a given direction by a single electron arrive at the detector at the same time. 
For many cases, both the plane wave and bandwidth-limited approximation are valid, which can reduce the computation time by 2 to 3 orders of magnitude. In this case

$$
\frac{d N_{s e}\left(\xi_{x e}, \xi_{y e}, \theta, \phi, \omega_{s}\right)}{d \Omega d \omega_{s}}=\int F_{\omega 0}(t) d t \sqrt{\frac{2}{\pi}} \frac{\frac{d \sigma}{d \Omega}\left(\xi_{x e}, \xi_{y e}, \theta, \phi\right)}{\Delta \omega_{0}\left|g\left(\xi_{x e}, \xi_{y e}, \theta\right)\right|} \exp \left\{-\frac{2\left[\frac{\omega_{s}}{g\left(\xi_{x e}, \xi_{y e}, \theta\right)}-\omega_{0}\right]^{2}}{\Delta \omega_{0}^{2}}\right\}
$$

Note that for the purposes of the output of the code, $\theta$ and $\phi$ are defined to be the angle specified by the transverse position of the detector and the distance of the detector from the interaction "point," which is defined to be the origin of the lab frame coordinate system. Because of the finite interaction size, this does not strictly correspond to the exact direction of all the scattered photons characterized to be at this observation angle. For most cases, however, the detector will be far away from the source (on the order of a meter), so the source size, which is usually less than $100 \mu \mathrm{m}$, will have a negligible effect on the observed spectrum.

As an illustrative example, we consider the case of a $50 \mathrm{MeV}(\gamma \approx 100)$ electron beam focused to an rms spot size of $20 \mu \mathrm{m}$ colliding head on with an $800 \mathrm{~nm}$ laser pulse in the plane-wave approximation. The laser pulse has a $1 / e^{2}$ pulse width of $1 \mathrm{ps}$, and the energy spread of the electron beam is assumed to be negligible. Figure 9 shows the calculated on-axis spectrum for the case of both $\varepsilon_{n x}=1 \mathrm{~mm} \operatorname{mrad}$ and $\varepsilon_{n x}=2 \mathrm{mmmrad}$, where $\varepsilon_{n x}$ is the normalized rms emittance of the electron bunch. For the $1 \mathrm{~mm}$ mrad case, the width of the x-ray spectrum is dominated by the laser bandwidth, and hence the shape of the spectrum is a symmetric Gaussian about the peak. For the $2 \mathrm{~mm}$ mrad example, the emittance of the beam is beginning to take over, characterized by the low energy tail in the distribution. Fig. 10 presents a more complete picture of the scattered $x$ rays. A false color image of the case of spectral energy density [energy $\left./\left(\operatorname{mrad}^{2} \mathrm{eV}\right)\right]$ is

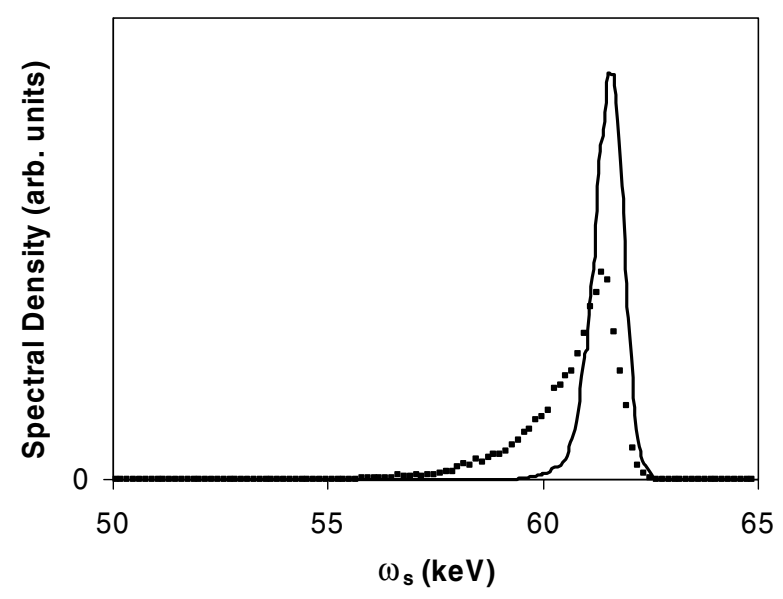

FIG. 9. On-axis $\mathrm{x}$-ray spectrum resulting from a head-on collision of a $50 \mathrm{MeV}$ electron beam with an $800 \mathrm{~nm}$ laser pulse for the case of an rms normalized emittance of $1 \mathrm{~mm} \mathrm{mrad}$ (line) and $2 \mathrm{~mm} \mathrm{mrad}$ (dots). plotted vs scattered x-ray energy, $\omega_{s}$, and divergence angle, $\theta$, in the plane perpendicular to the laser polarization. Note that the scattered spectrum is significantly narrower on axis. This fact, in conjunction with the larger number of photons scattered on-axis, leads to a much larger spectral brightness in this direction than in the off-axis directions.

\section{CHIRPED X-RAY PULSE PRODUCTION AND FEMTOSECOND PULSE SLICING}

The capability to provide complete temporal and spectral information of the $\mathrm{x}$-ray beam produced in a Thomson interaction makes the 3D time and frequencydomain code ideal for the study of chirped $\mathrm{x}$-ray pulse production. The production of chirped $\mathrm{x}$ rays would be of great benefit to ultrafast dynamic diffraction experiments, enabling fs $x$-ray pulse production. This can be accomplished through either pulse slicing or compression. Pulse slicing involves the selection of a small temporal fraction of the $\mathrm{x}$-ray pulse. With a chirped $\mathrm{x}$-ray pulse, this can be accomplished with either Bragg and/or multilayer monochromators [15]. The temporal portion of the $\mathrm{x}$-ray pulse that has a spectrum within the bandwidth of the monochromator will be selected, while the rest of the x-ray pulse will be discarded. Pulse compression, on the

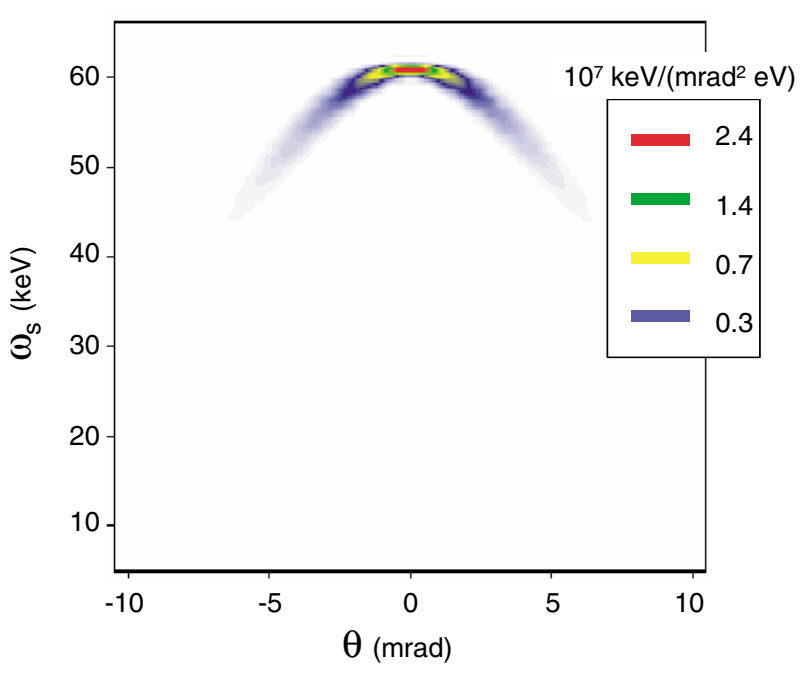

FIG. 10. (Color) False color plot of the spectral density of scattered $\mathrm{x}$ rays in the $y-z$ plane resulting from the head-on collision of a $50 \mathrm{MeV}$ electron bunch with $\varepsilon_{n x}=1 \mathrm{~mm} \mathrm{mrad}$ focused to an rms spot size of $20 \mu \mathrm{m}$ with an $800 \mathrm{~nm}, 0.5 \mathrm{ps}$ bandwidth laser pulse polarized in the $x$ direction. 


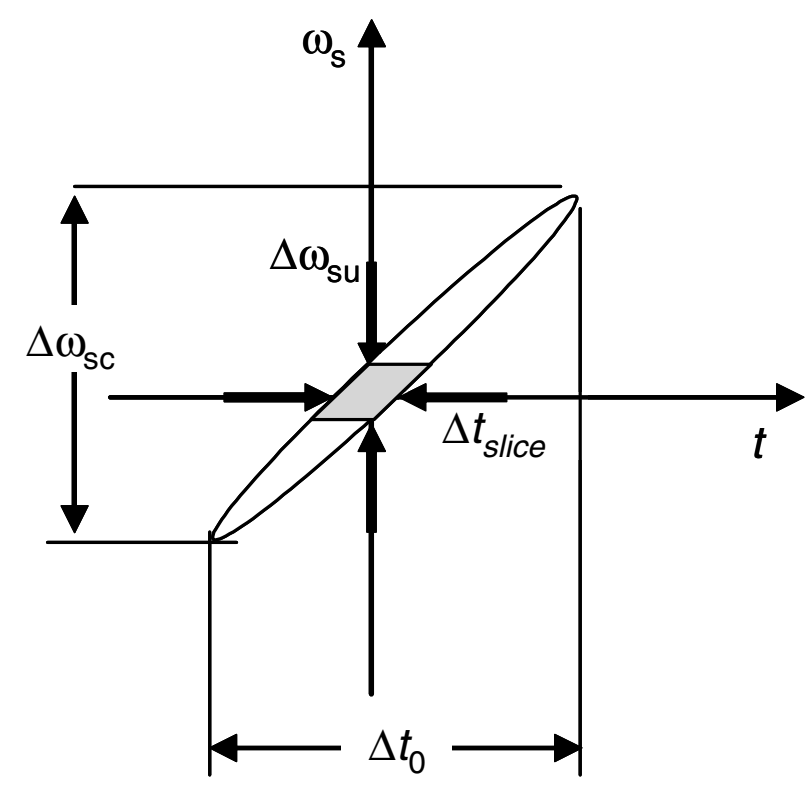

FIG. 11. Longitudinal phase space of a chirped x-ray pulse.

other hand, utilizes dispersive optics to cause photons of different wavelengths to travel different distances. If these distances are properly matched to the chirp in the $\mathrm{x}$-ray beam, the x-ray beam can be compressed, without necessarily discarding photons. Methods of compressing chirped x-ray pulses using strained crystals [16] and grating compressors [17] have been proposed. In addition to enabling ultrashort $\mathrm{x}$-ray pulse production, chirped $\mathrm{x}$-ray pulses could also be used to obtain a series of time-correlated measurements within a single Laue diffraction pattern, while eliminating the relevance of jitter between the pump and probe pulses [14].

The maximum temporal resolution provided by a chirped pulse is determined by the ratio of the uncorrelated spectral bandwidth $\Delta \omega_{s u}$, defined to be the minimum spectral width for a single temporal slice within the pulse, to the time integrated, or correlated, spectral bandwidth, $\Delta \omega_{s c}$ (see Fig. 1). While $\Delta \omega_{s u}$ results from the emittance of the electron beam, the bandwidth of the laser, and the uncorrelated energy spread of the electron beam, $\Delta \omega_{s c}$ is determined by the larger time integrated energy spread of the electron bunch resulting from the energy chirp. For the case where $\Delta \omega_{s c} \gg \Delta \omega_{s u}, \Delta \omega_{s c}$ can be roughly equated with the total time integrated energy spread of the x-ray beam, as depicted in Fig. 11. In this case, the minimum $x$-ray pulse length achievable through slicing or compression, $\Delta t_{\text {slice, }}$, will be given by

$$
\Delta t_{\text {slice }} \sim \frac{\Delta \omega_{s u}}{\Delta \omega_{s c}} \Delta t_{0}
$$

where $\Delta t_{0}$ is the $1 / e^{2}$ intensity width of the total x-ray pulse.

Two possible methods for producing chirped x-ray pulses through Thomson scattering are (1) preparing an energy chirped electron bunch, and (2) preparing a chirped laser pulse (Fig. 12). In the first method, the electron bunch can be chirped by passing it through an accelerator section near the zero crossing of the rf wave. In this case, the average energy gain through the section will be zero, but a linear correlation between time and energy will be induced in the electron bunch. Subsequently, when the electron bunch collides with the laser pulse, the $\mathrm{x}$ rays, which are automatically time correlated with the electrons, will also receive a chirp. Since the scattered photon energy scales as the square of the electron beam energy, the correlated energy spread of the $\mathrm{x}$ rays, $\Delta \omega_{s c}$, will be given by

$$
\frac{\Delta \omega_{s c}}{\bar{\omega}_{s}} \sim 2 \frac{\Delta \gamma_{c}}{\bar{\gamma}}
$$

where $\bar{\gamma}$ is the average electron beam relativistic Lorentz factor, $\bar{\omega}_{s}$ is the average scattered photon energy at a given observation direction, and $\Delta \gamma_{c}$ is the correlated energy spread of the electron bunch.

For the second case (chirping of the incident laser pulse), $\Delta \omega_{s c}$, will depend on the interaction geometry. For a head-on collision, each electron will sample the entire laser pulse (assuming the laser pulse is short compared to the Rayleigh range), and hence will radiate the entire Doppler up-shifted spectrum of the laser pulse. However, if the interaction occurs at $90^{\circ}$, different electrons will sample different temporal positions within the laser pulse, and if the laser pulse is chirped, will radiate different spectra. To maximize this effect, the width of

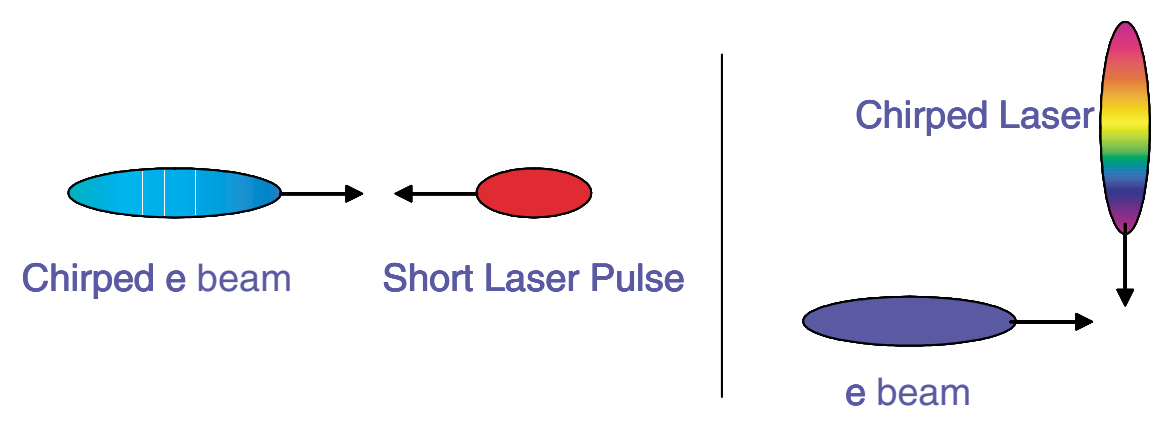

FIG. 12. (Color) Methods for chirped x-ray pulse production. 
the laser focus should be small compared to its duration so as to minimize the longitudinal range sampled by each electron in the bunch. However, the spectral broadening due to the laser focus becomes more significant in a sideon collision [see Sec. IV B], which will limit how small the interaction spot size can be without significantly increasing the uncorrelated spectral width of the scattered $\mathrm{x}$ rays.

\section{A. Chirped x-ray production with an energy chirped electron bunch}

To produce a chirped electron beam, the electron bunch is first accelerated to relativistic energies in an rf accelerator. Subsequently, the bunch, with pulse duration specified in terms of $\mathrm{rf}$ degrees, $\Delta \varphi_{e}$, is injected at the zero crossing of the rf wave in a later accelerator section. This will impart a correlation between the energy of the electrons and the phase, which corresponds directly with time. Since the electron bunch is already relativistic, the output pulse length will be largely unaltered from the input pulse length, resulting in a chirped electron bunch. If the maximum energy gain by the accelerator section is represented by $\Delta \gamma_{m}$, and the input electron beam energy is represented by $\bar{\gamma}$, then the fractional correlated energy spread at the output of the accelerator section will be given by

$$
\frac{\Delta \gamma_{c}}{\bar{\gamma}} \sim \frac{\Delta \gamma_{m}}{\bar{\gamma}} \Delta \varphi_{e}
$$

where $\Delta \varphi_{e}$ is typically a few rf degrees. If we assume a typical accelerating gradient of $10 \mathrm{MV} / \mathrm{m}$ for a standard $S$-band $(2.85 \mathrm{GHz})$ accelerator, a chirp of $0.17 \mathrm{MeV}$ per meter of accelerator per picosecond of electron bunch duration will be achieved.

Ideally, the electron beam energy chirp expressed in Eq. (71) will result in the corresponding $x$-ray energy chirp expressed in Eq. (70). For this to actually be achieved, however, the competing spectral broadening effects resulting from the uncorrelated energy spread of the electron beam, the laser pulse bandwidth, and the electron beam emittance must be minimized [see Eq. (63)]. It can be expected that the maximum reasonable value for $\Delta \gamma_{c}$ will only be a few percent of the average electron beam energy due to constraints of the chromaticity of the final focus optics, as well as the limited accelerator length available to perform the chirping. Thus, to maximize the $\mathrm{x}$-ray beam chirp, which will be characterized by the ratio of the correlated $x$-ray spectral width, $\Delta \omega_{s c}$, to the uncorrelated spectral width $\Delta \omega_{s u}$, these competing spectral broadening effects should be much less than $1 \%$, i.e.,

$$
\frac{\Delta \omega_{s u}}{\bar{\omega}_{s}} \ll \frac{\Delta \omega_{s c}}{\bar{\omega}_{s}} \sim 1 \% .
$$

The uncorrelated electron beam energy spread is typically less than $0.1 \%$, and the broadening due to the laser bandwidth should meet the requirement of Eq. (72), provided the bandwidth-limited pulse length is longer than about $1 \mathrm{ps}$, which is still within the Rayleigh range of a typical laser focus. Thus, the primary challenge to satisfying Eq. (72) is the minimization of the spectral broadening due to the electron beam emittance. In order for this to be achieved, the transverse divergence of the electron beam at the interaction point, $\Delta \xi_{x e}$, must be much less than overall $\mathrm{x}$-ray beam divergence, given approximately by $1 / \gamma$, or, using Eqs. (62) and (72),

$$
\Delta \xi_{x e}^{2} \ll \frac{2}{\gamma^{2}} \frac{\Delta \omega_{s c}}{\bar{\omega}_{s}} .
$$

Assuming an electron beam of energy $50 \mathrm{MeV}$ with a $1 \%$ rms energy chirp, an rms divergence angle of much less than $2 \mathrm{mrad}$ will be required to achieve adequate spectral sharpness to resolve the x-ray chirp. In terms of the normalized rms emittance and the final focus spot size, Eq. (72) becomes

$$
\frac{\Delta \omega_{s u}}{\bar{\omega}_{\mathrm{s}}} \approx \frac{2 \varepsilon_{n x}^{2}}{\sigma_{x f}^{2}} \ll \frac{\Delta \omega_{s c}}{\bar{\omega}_{s}},
$$

where $\sigma_{x f}$ is the rms size in $x$ of the electron beam focus. Assuming an rms spot size of less than $50 \mu \mathrm{m}$ is desired for the Thomson interaction, it is seen from Eq. (62) that an rms normalized emittance of much less than $10 \mathrm{~mm}$ mrad will be required. While challenging, the electron bunch parameters required to produce a highly correlated x-ray spectrum should be achievable. For example, electron beam emittances as low as $1 \mathrm{~mm}$ mrad with bunch charges of several hundred pico-Coulombs have been demonstrated at Brookhaven National Laboratory [28].

For the calculations presented here, the particle dynamics code PARMELA was used to simulate the electron bunch production at the PLEIADES facility at LLNL [11]. The accelerator consists of several major components, including an $S$-band photocathode rf gun and four $2.5 \mathrm{~m}$ $S$-band accelerator sections. The accelerator sections run at gradients up to $10 \mathrm{MV} / \mathrm{m}$. In the simulation, the electron beam is accelerated through the first three sections up to an average energy of $50 \mathrm{MeV}$, while the last section is used to induce an energy chirp in the electron beam by injecting $90^{\circ}$ ahead of the rf crest. In addition, for this particular case the electron beam was also injected near the zero crossing in the first accelerator section, resulting in additional chirping and some longitudinal compression of the bunch. The longitudinal phase space and time integrated transverse phase space of the simulated electron beam at the interaction location are shown in Fig. 13. The rms bunch length is $0.7 \mathrm{ps}$, and the correlated rms energy spread is $1.3 \%$, while the uncorrelated energy spread is $0.014 \%$. The normalized rms emittance is $0.7 \mathrm{~mm}$ mrad. The electron beam focus was simulated using a $25 \mathrm{~cm}$ focal length quadrupole triplet, indicating the beam could be focused to an rms spot size of $35 \mu \mathrm{m}$ 

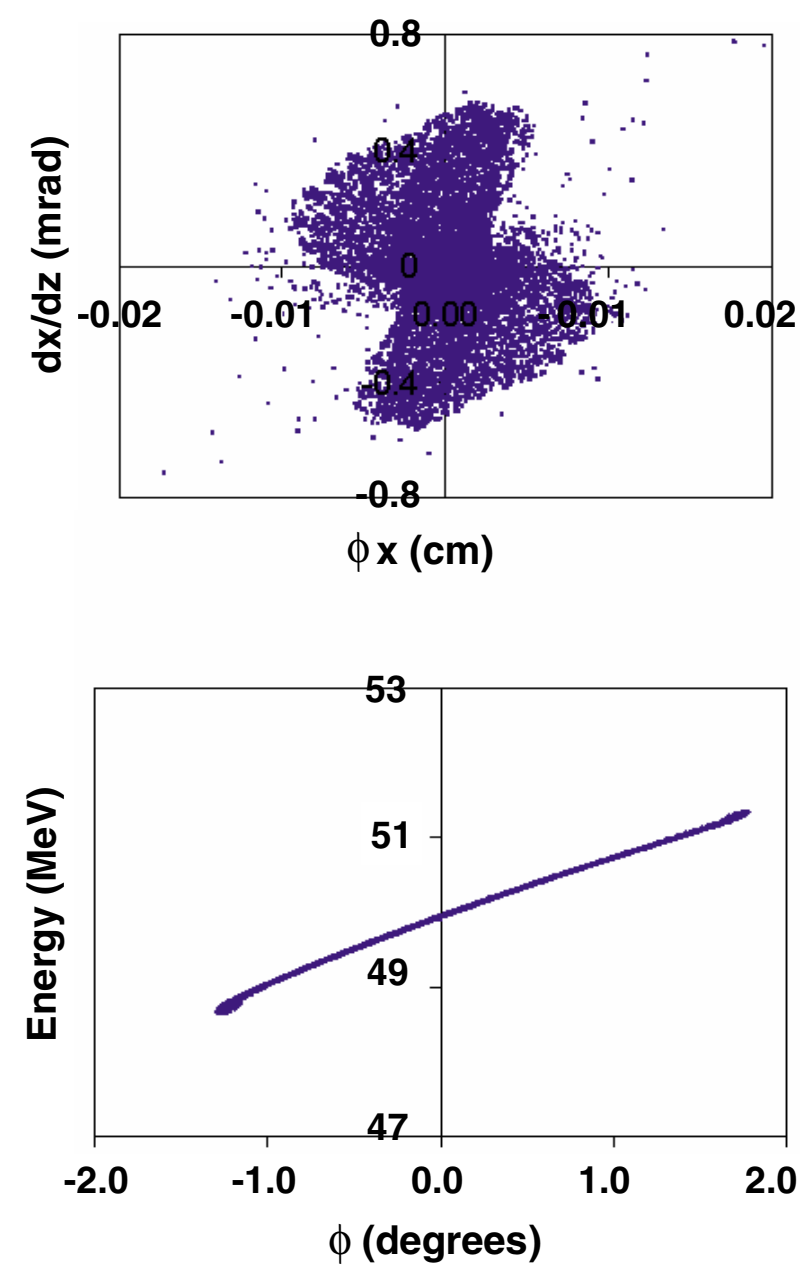

FIG. 13. (Color) Simulated transverse and longitudinal phase space of the electron beam at the interaction point.

with an rms divergence angle of only $0.25 \mathrm{mrad}$. The electron beam parameters are summarized in Table I

To simulate the production of Thomson $\mathrm{x}$ rays, the macro-particle coordinates from the PARMELA simulation were loaded into the time and frequency-domain Thomson code. The interaction geometry was taken to be that of a head-on collision, with the laser pulse having a $1 / e^{2}$ intensity pulse duration of $0.5 \mathrm{ps}$, and focused to a

TABLE I. Summary of the simulated electron beam parameters at the interaction.

\begin{tabular}{ccc}
\hline \hline Parameter & Description & Value \\
\hline$E$ & Average beam energy & $50 \mathrm{MeV}$ \\
$Q$ & Bunch charge & $0.1 \mathrm{nC}$ \\
$\varepsilon_{n x}$ & rms normalized emittance & $0.7 \mathrm{~mm} \mathrm{mrad}$ \\
$\sigma_{x f}$ & rms spot size & $35 \mu \mathrm{m}$ \\
$\sigma_{t}$ & rms duration & $0.7 \mathrm{ps}$ \\
$\Delta \gamma_{c}$ & $1 / e^{2}$ correlated energy spread & $2.6 \%$ \\
$\Delta \gamma_{u}$ & Uncorrelated energy spread & $0.028 \%$ \\
$\Delta \xi_{x e}$ & $1 / e^{2}$ divergence & $0.5 \mathrm{mrad}$ \\
\hline \hline
\end{tabular}
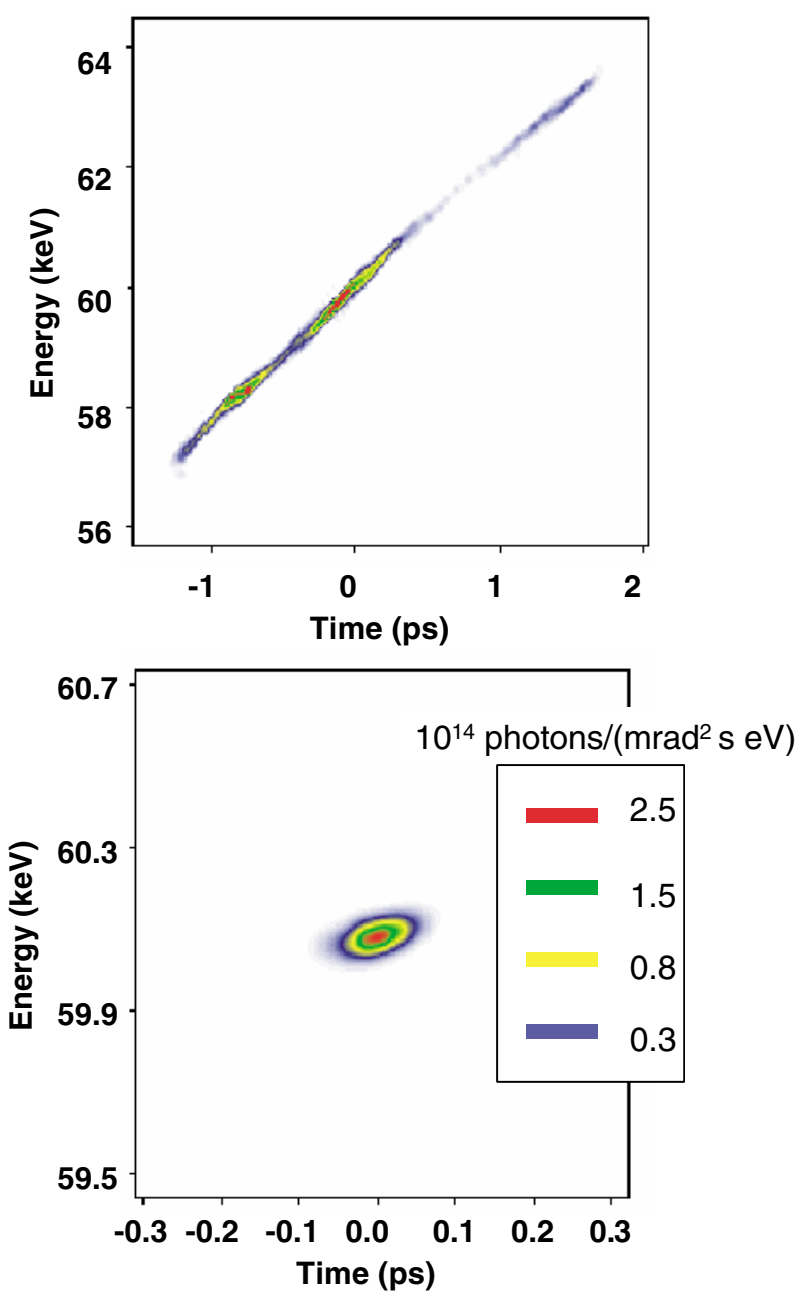

FIG. 14. (Color) Simulated on-axis $\mathrm{x}$-ray spectrum of the chirped Thomson scattered $\mathrm{x}$ rays before (top panel), and after (bottom panel) spectral filtering.

$1 / e^{2}$ radius of $36 \mu \mathrm{m}$. Because of the insensitivity of the $\mathrm{x}$-ray spectrum to the laser focus parameters in the headon geometry, the plane-wave approximation was invoked in the calculation to speed up computation time.

The results of the calculations are summarized in Table II. The total $\mathrm{x}$-ray dose is about $10^{7}$ photons, with an average on-axis $x$-ray energy equal to about $60 \mathrm{keV}$. The calculated on-axis $x$-ray spectrum is shown in Fig. 14. It is seen that excellent time/energy correlation is present, with a ratio of the correlated to uncorrelated spectral width equal to about 20:1. The longitudinal structure in the $\mathrm{x}$-ray intensity is due to the longitudinal dependence of the focal spot position within the electron bunch.

Also shown in Fig. 14 is the result of a simulation of pulse slicing by spectral filtering of the $\mathrm{x}$-ray pulse. This can be accomplished through Bragg reflection with a bent crystal monochromator. This process was simulated by multiplying the scattered intensity at each wavelength by a Lorentzian attenuation factor with a FWHM 
TABLE II. Chirped Thomson $\mathrm{x}$-ray beam parameters.

\begin{tabular}{ccc}
\hline \hline Parameter & Description & Value \\
\hline$N_{s}$ & Total x-ray dose & $10^{7}$ \\
$\omega_{s}(\theta=0)$ & On axis x-ray energy & $60 \mathrm{keV}$ \\
$\Delta \omega_{s c}$ & $1 / e^{2}$ correlated spectral width & $4.9 \%$ \\
$\Delta \omega_{s u}$ & Uncorrelated spectral width & $0.32 \%$ \\
$\Delta t_{s}$ & $1 / e^{2}$ duration & $1.4 \mathrm{ps}$ \\
$\Delta t_{f}$ & Minimum sliced duration & $70 \mathrm{fs}$ \\
$N_{s f}$ & Photons in sliced pulse $(0.1 \mathrm{mrad})$ & $\sim 20$ \\
$\Delta w_{\gamma}$ & $1 / e^{2}$ source size & $36 \mu \mathrm{m}$ \\
$B$ & Brightness of source & $10^{19} \mathrm{ph} . /\left(\mathrm{s} \mathrm{mm}^{2} \mathrm{mrad}^{2} 0.1 \%\right.$ b.w. $)$ \\
\hline \hline
\end{tabular}

of $0.1 \%$ about the center frequency of $60 \mathrm{keV}$. This corresponds roughly to a Bragg reflection from a LiF crystal (200) tilted at $3.0^{\circ}$ to the $\mathrm{x}$-ray beam direction. The resulting FWHM pulse duration for the filtered $x$-ray pulse, assuming sufficient radial collimation, is only about $70 \mathrm{fs}$, about a factor of 20 down from the initial duration of $1.4 \mathrm{ps}$. Note that the increase in uncorrelated spectral width due to the finite radial collimation of the $\mathrm{X}$-ray beam scales as roughly $\left(\gamma \theta_{r}\right)^{2} / 2$, where $\theta_{r}$ is the angular half-width of the collimation cone. For the example under consideration, where $\Delta \omega_{s u} / \omega_{s}$ is equal to $0.3 \%$, and $\gamma$ is about 100 , the radially integrated spectrum should be about the same as the on-axis spectrum for $\theta_{r}<0.5 \mathrm{mrad}$, implying only a modest increase in the duration of the sliced $\mathrm{x}$-ray pulse. This effect is illustrated in Fig. 15.

The collimating and slicing process required to produce the short, monochromatic $\mathrm{x}$-ray pulses will typically reduce the number of photons in the $x$-ray pulse by a factor of about $10^{4}-10^{5}$, depending on the percentage acceptance of the slicing optic used (typically $10^{-3}-10^{-4}$ ) and the acceptable radial width of the collimation. For the cases studied above, the photon dose will vary from approximately $10^{1}-10^{3}$ depending on the initial collimation imposed on the $\mathrm{x}$-ray pulse, highlighting the inherent inefficiency of the pulse slicing method. However, due to the extremely small pulse duration, low angular divergence, and narrow bandwidth, the brightness of the source is still very high, about $10^{19}$ photons $/\left(\mathrm{s} \mathrm{mm}^{2} \mathrm{mrad}^{2} 0.1 \%\right.$ b.w. $)$. More efficient methods of pulse compression, like pulse compressions with strained crystals [16] or grating compressors [17], could potentially lead to even higher brightness $\mathrm{x}$-ray pulses.

\section{B. Simulation of chirped $x$-ray production with a chirped laser pulse}

Chirped $x$ rays can also be produced by colliding an electron bunch with a chirped laser pulse. This can be performed in a side-on $\left(\theta_{0}=90^{\circ}\right)$ collision geometry, in which case the portion of the laser pulse seen by each electron will depend on the longitudinal position within the electron bunch. The minimum spectral width radiated by each electron will be determined by the slice bandwidth of the chirped laser pulse, defined to be the bandwidth at a given longitudinal position in the pulse, given approximately by

$$
\frac{\Delta \omega_{0 u}}{\Delta \omega_{0}} \approx \frac{\Delta t_{\min }}{\Delta t_{c}}=\frac{2}{\Delta \omega_{0} \Delta t_{c}},
$$

where $\Delta \omega_{0}$ is the total bandwidth of the laser pulse, $\Delta t_{\text {min }}$ is the bandwidth-limited pulse length, $\Delta t_{c}$ is the chirped pulse length, and $\Delta \omega_{0 u}$ is the slice (or uncorrelated)
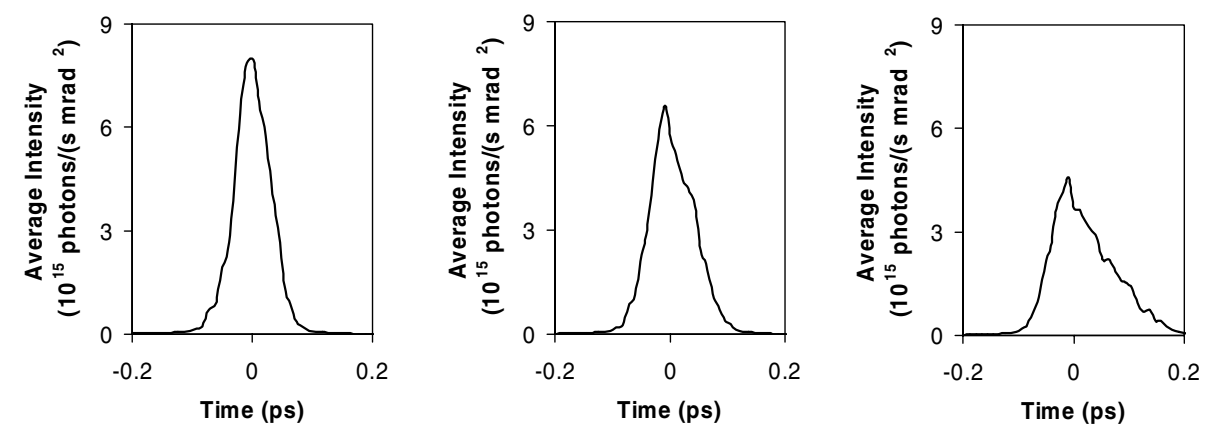

FIG. 15. Simulation of pulse slicing by Bragg reflection showing the spatially averaged intensity vs time for the case of radial collimation widths of $0.1 \mathrm{mrad}$ (left panel), $0.25 \mathrm{mrad}$ (middle panel), and $0.5 \mathrm{mrad}$ (right panel), corresponding to FWHM pulse durations of 70, 80, and $100 \mathrm{fs}$, respectively, and total photon counts of 19, 113, and 420 . 
bandwidth of the laser pulse. For cases where the chirped pulse length is much greater than the bandwidth-limited pulse length, the center frequency as a function of time, $t$, within the laser pulse can be expressed as

$$
\omega_{0}(t) \approx \omega_{0} \pm \frac{t}{\Delta t_{c}} \Delta \omega_{0},
$$

where the sign indicates a positive or negative chirp. The fractional bandwidth seen by each slice of the electron bunch will be proportional to the ratio of the laser beam waist to the bunch length. Thus, the larger the focal spot of the laser, the larger the uncorrelated spectral width of the scattered $\mathrm{x}$-ray pulses. However, if the focal spot becomes too small, the spectral broadening due to the perpendicular $k$ vectors in the laser focus will become significant (see Sec. IV B), which will place a limit on how small the laser focus can be made. The total uncorrelated width of the scattered $\mathrm{x}$-ray spectrum, neglecting broadening effects of the electron beam, can be estimated to be

$$
\frac{\Delta \omega_{s u}}{\bar{\omega} \bar{s}_{\mathrm{s}}} \approx \sqrt{\left(\frac{\lambda_{0}}{\pi c \Delta t_{\min }}\right)^{2}\left(\frac{w_{0}}{c \Delta t_{c}}\right)^{2}+\left(\frac{\lambda_{0}}{\pi w_{0}}\right)^{2}+\left(\frac{\lambda_{0}}{\pi c \Delta t_{c}}\right)^{2}},
$$

where $w_{0}$ is the $1 / e^{2}$ intensity radius of the laser pulse at the focus, and $\Delta \omega_{s u}$ is the uncorrelated bandwidth of the $x$ rays scattered by a single electron. The first term in Eq. (77) represents the broadening due to the transit of the electron through the finite laser focus spot, the second term is the broadening from $\Delta k_{\perp}$, while the last term represents $\Delta \omega_{0 u}$. In addition to minimizing $\Delta \omega_{s u}$, in order to maximize the total $\mathrm{x}$-ray dose it will be necessary to limit $\Delta t_{c}$ to about the same pulse duration as the electron bunch. Combining these three effects places relatively severe limitations on the ability to produce a chirped $\mathrm{x}$-ray pulse. The minimum sliced pulse duration can be estimated from Eqs. (69) and (77) to be

$$
\frac{\Delta t_{\text {slice }}}{\Delta t_{0}} \approx \sqrt{\left(\frac{w_{0}}{c \Delta t_{c}}\right)^{2}+\left(\frac{\Delta t_{\min } c}{w_{0}}\right)^{2}+\left(\frac{\Delta t_{\min }}{\Delta t_{c}}\right)^{2}},
$$

where it has been assumed that the laser pulse duration is less than or equal to the electron bunch duration, and hence, the total $\mathrm{x}$-ray bandwidth radiated by the electron beam is comparable to the Doppler up-shifted bandwidth of the laser pulse. Note that Eq. (78) represents the minimum sliced pulse duration assuming a zero emittance electron beam with zero spot size at the interaction. In reality, the sliced pulse duration will be larger than that specified by Eq. (78), since the uncorrelated bandwidth of the scattered $\mathrm{x}$-ray pulse will be increased by both the electron beam emittance and the energy spread. In addition, the finite spot size of the electron beam will result in electrons within the same longitudinal slice of the electron bunch sampling different regions of the incident laser spectrum, ultimately leading to a larger uncorrelated bandwidth of the x-ray pulse, and hence, longer minimum pulse duration.

We consider a 100 fs bandwidth-limited laser pulse stretched to a $1 / e^{2}$ pulse length of $1.5 \mathrm{ps}$. The pulse is taken to collide side on with a $50 \mathrm{MeV}$ electron beam of identical pulse width, emittance, and focal spot as previously considered (Table I), though in this case the electron bunch is not chirped. To minimize the spectral broadening described by Eqs. (77) and (78), the $1 / e^{2}$ spot size of the laser focus was chosen to be $100 \mu \mathrm{m}$. After simulations with several spot sizes, this was determined to be close to the optimum for minimizing the sliced pulse duration. Figure 16 shows the on-axis time resolved $\mathrm{X}$-ray spectrum before and after pulse slicing. While a clear correlation between the $\mathrm{x}$-ray energy and time is apparent, the uncorrelated spectral width is much more significant in this case than with the previous example. As a result, the effectiveness of pulse slicing through spectral filtering is diminished, with only a modest shortening of the rms pulse duration from about 0.53 to $0.33 \mathrm{ps}$. Additionally, both the photon flux and the brightness
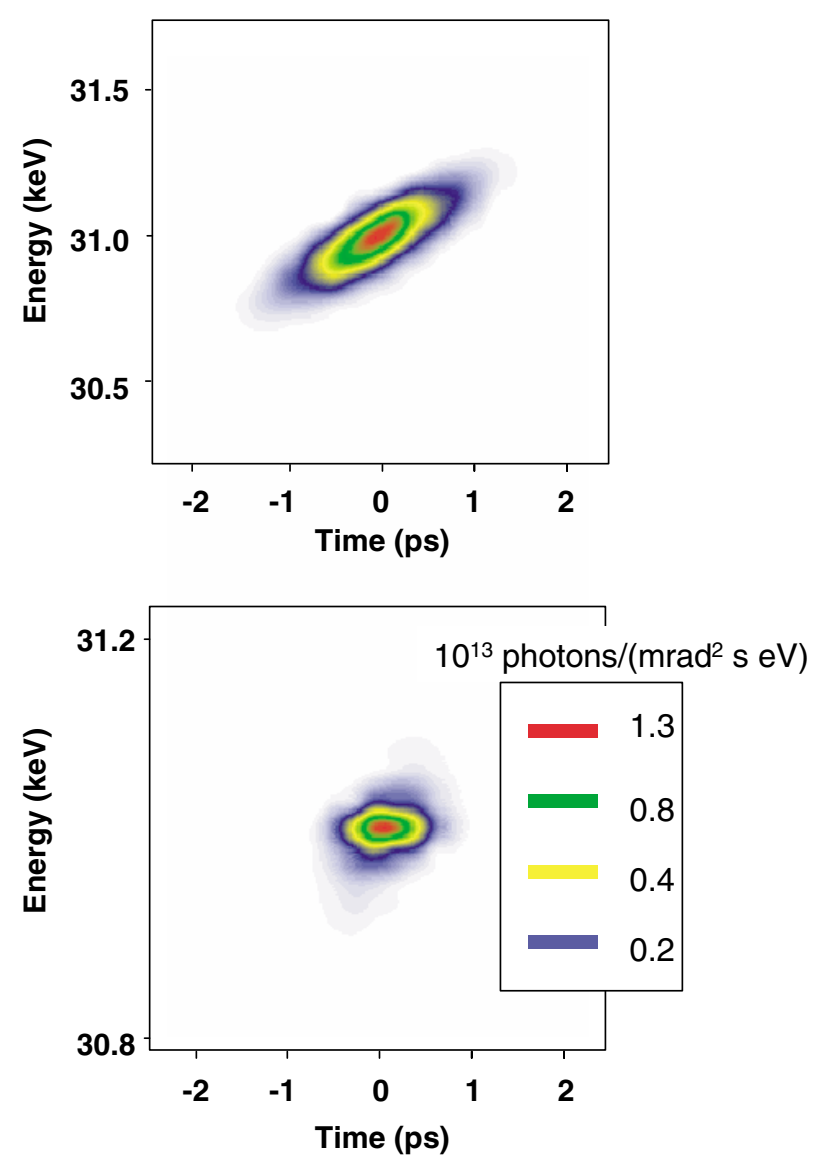

FIG. 16. (Color) Simulated on-axis $\mathrm{x}$-ray spectrum for the chirped Thomson scattered $\mathrm{x}$ rays produced by colliding a chirped laser pulse side on with an unchirped electron bunch, before (top panel) and after (bottom panel) spectral filtering. 
are decreased from the previous example by about a factor of 20 .

It should be noted that further optimization of the interaction parameters can lead to a smaller pulse duration than shown in the example here. By stretching a laser pulse with a larger bandwidth, the correlated spectral bandwidth in the resulting $x$-ray pulse will be proportionally larger, potentially resulting in a shorter sliced $x$-ray pulse. However, the finite spot size of the electron bunch, which is limited by the electron beam emittance, will place a limit on the minimum uncorrelated bandwidth of the x-ray pulse, essentially limiting the minimum sliced pulse duration to the time of flight across the electron beam waist. In addition, the larger correlated bandwidth of the $x$ rays will result in a smaller number of photons in the sliced $x$-ray pulse. If we reconsider the case of the $1.5 \mathrm{ps}$ stretched laser pulse discussed above assuming a 25 fs bandwidth-limited pulse length, we find the optimum $1 / e^{2}$ laser spot size to be about $50 \mu \mathrm{m}$, resulting in a minimum sliced pulse duration of about $0.21 \mathrm{ps}$. This is a $35 \%$ reduction in pulse duration from the case involving the $100 \mathrm{fs}$ bandwidth-limited pulse, but the total photon flux is also reduced by more than half.

Based on the cases discussed above, it is apparent that colliding a chirped electron beam with a laser pulse is a significantly more effective method for producing chirped x-ray pulses for ultrafast pulse slicing and/or compression than utilizing a chirped laser pulse. However, it is seen that a moderate time/energy correlation can be achieved by the use of a chirped laser pulse in a side-on geometry. In some circumstances this may be useful for providing moderate pulse slicing or compression of Thomson scattered x-ray pulses. For example, when the electron bunch is sub-ps in duration, energy chirping may not be practical, and the use of a chirped laser pulse may be the most beneficial approach for producing a chirped x-ray pulse.

\section{CONCLUSIONS}

The development of a femtosecond, hard x-ray source capable of probing inner-shell electron properties on atomic time scales would open up regions of currently underexplored science, such as phase transitions in materials under shock loading and chemical reaction dynamics. Thomson backscattering of an intense laser pulse with a high-brightness electron beam is a promising means of meeting the demanding specifications of such an $x$-ray source. In this paper, a recently developed 3D time and frequency-domain code for simulations of linear Thomson scattering for arbitrary interaction geometries has been applied to the study of chirped x-ray pulse production and ultrafast pulse slicing. The code employs a generalized relativistic Thomson differential cross section and was designed to enhance existing computational capabilities. It will be beneficial for both the design of
Thomson scattered x-ray sources, as well as future experiments and applications utilizing such sources. In particular, the new code is well suited for analyzing time-dependent spectra of the $\mathrm{x}$ rays produced from linear Thomson scattering, and hence is ideally suited for the study of chirped x-ray pulse production. Spectral broadening of the scattered $x$-ray pulse resulting from the incident laser bandwidth, laser focus, and the transverse and longitudinal phase space of the electron beam were examined. While chirped x-ray pulse production using both a chirped electron beam and a chirped laser pulse were presented, it was shown that the most promising method for producing chirped $\mathrm{x}$ rays capable of sup $100 \mathrm{fs}$ time resolution involved the used of an energy chirped electron beam at the interaction. The primary limitation on the ability to produce chirped $\mathrm{x}$-ray pulses using this method is the uncorrelated spectral broadening due to the electron beam emittance. Generally, it can be expected that the normalized rms emittance of the electron bunch should be on the order of $1 \mathrm{~mm}$ mrad if both a welldefined temporal $\mathrm{x}$-ray energy correlation and a sizable photon flux are desirable. Simulations of the x-ray production from a collision of an energy chirped, $50 \mathrm{MeV}, 0.1 \mathrm{nC}, 0.7 \mathrm{~mm}$ mrad electron bunch, and an $800 \mathrm{~nm}, 500 \mathrm{~mJ}$ laser pulse resulted in an x-ray pulse with excellent temporal energy correlation, with a minimum sliced pulse duration of about $70 \mathrm{fs}$ FWHM, and a peak spectral brightness in excess of $10^{19}$ photons $/\left(\mathrm{s} \mathrm{mm}^{2} \mathrm{mrad}^{2} 0.1 \%\right.$ b.w. $)$. Such an $\mathrm{x}$-ray source would provide significant improvement over the time resolution provided by other tunable, hard x-ray sources. While the total photon dose resulting from the slicing process is modest, the application of $\mathrm{x}$-ray pulse compression schemes could lead to a much larger peak $\mathrm{x}$ ray flux. Additionally, it is conceivable that a sliced x-ray pulse from a Thomson source could be used to seed and $x$ ray FEL, in congruence with proposed two-stage FEL schemes. Finally, we note that the theory and computer code described in this paper have been benchmarked against experimental data obtained at LLNL's PLEIADES facility. These results are described in detail in a companion paper.

\section{ACKNOWLEDGMENTS}

This work was performed under the auspices of the U.S. Department of Energy by the University of California, Lawrence Livermore National Laboratory under Contract No. W-7405-Eng-48. UCRL-JP-200521.

[1] A. H. Chin et al., Phys. Rev. Lett. 83, 336 (1999).

[2] A. M. Lindenberg et al., Phys. Rev. Lett. 84, 111 (2000).

[3] J. N. Galayda, in Proceedings of the 8th International Conference on X-Ray Lasers, Aspen, CO, 2002 (AIP, Aspen, 2002), p. 365. 
[4] R.W. Schoenlein et al., Science 274, 236 (1996).

[5] W. P. Leemans et al., Phys. Rev. Lett. 77, 4182 (1996).

[6] S. Kashiwagi et al., Nucl. Instrum. Methods Phys. Res., Sect. A 455, 36 (2000).

[7] I.V. Pogorelsky et al., AIP Conf. Proc. 569, 571 (2001).

[8] A. E. Vlieks, G. Caryotakis, W. R. Fowkes, E. N. Jongewaard, E. C. Landahl, R. Loewen, and N.C. Luhmann, AIP Conf. Proc. 625, 107 (2002).

[9] R. P. Fischer, A. Ting, C. I. Moore, P. Sprangle, M. Baine, R. Elton, and S. Ride, in Proceedings of the 2001 Particle Accelerator Conference, Chicago, IL (IEEE, Piscataway, 2001), p. 2644.

[10] A. Fukasawa et al., Int. J. Appl. Electromag. Mech. 14, 221 (2001).

[11] D. Gibson et al., Phys. Plasmas 11, 2857 (2004).

[12] T. Guo, C. H. Spielmann, B. C. Walker, and C. P. J. Barty, Rev. Sci. Instrum. 72, 41 (2001).

[13] J. Arthur, G. Materlik, R. Tatchyn, and H. Winick, Rev. Sci. Instrum. 66, 1987 (1995).

[14] K. Moffat, Faraday Discuss. Chem. Soc. 122, 65 (2002).

[15] C. B. Schroeder et al., Nucl. Instrum. Methods Phys. Res., Sect. A 483, 89 (2002).

[16] H. N. Chapman and K. A. Nugent, Opt. Commun. 205, 351 (2002).
[17] C. Pellegrini, Nucl. Instrum. Methods Phys. Res., Sect. A 445, 124 (2000).

[18] F.V. Hartemann et al., Phys. Rev. E 64, 016501 (2001).

[19] G. A. Krafft, in Proceedings of the 1997 Particle Accelerator Conference, Vancouver, Canada (IEEE, Piscataway, NJ, 1998), Vol. 1, p. 739.

[20] W. P. Leemans et al., IEEE J. Quantum Electron. 33, 1925 (1997).

[21] S. K. Ride, E. Esarey, and M. Baine, Phys. Rev. E 52, 5425 (1995).

[22] F. V. Hartemann and A. K. Kerman, Phys. Rev. Lett. 76, 624 (1996).

[23] E. Esarey, S. K. Ride, and P. Sprangle, Phys. Rev. E 48, 3003 (1993).

[24] C. Bula et al., Phys. Rev. Lett. 76, 3116 (1996).

[25] J. D. Jackson, Classical Electrodynamics (John Wiley \& Sons, New York, 1975), 2nd ed.

[26] J. B. Rosenzweig, Fundamentals of Beam Physics (Oxford University Press, New York, 2003).

[27] F.V. Hartemann, High-Field Electrodynamics (CRC Press, Boca Raton, FL, 2002) p. 453.

[28] V. Yakimenko et al., Nucl. Instrum. Methods Phys. Res., Sect. A 483, 277 (2002). 